\title{
Stabilization of nonclassical states of one- and two-mode radiation fields by reservoir engineering
}

\author{
A. Sarlette, ${ }^{1, *}$ Z. Leghtas, ${ }^{2}$ M. Brune, ${ }^{3}$ J.M. Raimond, ${ }^{3}$ and P. Rouchon ${ }^{4}$ \\ ${ }^{1}$ SYSTeMS, Ghent University, Technologiepark 914, 9052 Zwijnaarde, Belgium \\ ${ }^{2}$ INRIA Paris-Rocquencourt, Domaine de Voluceau, \\ Rocquencourt B.P. 105, 78153 Le Chesnay Cedex, France \\ ${ }^{3}$ Laboratoire Kastler-Brossel, ENS, UMPC-Paris 6, CNRS, 24 rue Lhomond, 75005 Paris, France \\ ${ }^{4}$ Centre Automatique et Systèmes, Mines ParisTech, 60 boulevard Saint Michel, 75006 Paris, France
}

(Dated: June 1, 2012)

\begin{abstract}
We analyze a quantum reservoir engineering method, originally introduced by Sarlette et al [Phys. Rev. Lett. 107, 010402 (2011)], for the stabilization of non-classical field states in high quality cavities. We generalize the method to the protection of mesoscopic entangled field states shared by two non-degenerate field modes. The reservoir consists of a stream of atoms consecutively interacting with the cavity. Each individual atom-cavity interaction follows the same time-varying Hamiltonian, combining resonant with non-resonant parts. We get a detailed insight into the competition between the engineered reservoir and decoherence. We show that the operation is quite insensitive to experimental imperfections and that it could thus be implemented in the near future, either in the context of microwave Cavity Quantum Electrodynamics or in that of circuit-QED.
\end{abstract}

PACS numbers: 03.65.Yz, 03.67.Pp, 42.50.Pq

\section{INTRODUCTION}

Nonclassical electromagnetic field states are extremely important, both for a fundamental understanding of the quantum properties of light and for their possible use in practical applications. For instance, fluctuations on one quadrature can be brought below those of the vacuum state or below those of a classical coherent state, by resorting to squeezed states (SS) [1]. They lead to interesting methods for high-precision measurements and metrology [2]. Among others, they are considered for reducing the noise of gravitational wave interferometers below the standard quantum limit [3].

Mesoscopic field state superpositions (MFSS) are also the focus of an intense interest. They involve a quantum superposition of two quasi-classical coherent components with different complex amplitudes. These counterintuitive states bridge the gap between the quantum and the classical worlds and shed light onto the decoherence process responsible for the conspicuous lack of superpositions at our scale [4].

Finally, entangled superpositions of mesoscopic states (ESMS) shared by several field modes are even more intriguing. They violate generalized Bell inequalities [5], illustrating the non-local nature of quantum physics. However, their non-local character is rapidly lost in a fast decoherence process [6], driving them back into a statistical mixture that can be understood in terms of a classical local hidden variable model. This interplay of decoherence and non-locality opens fascinating perspectives for exploring the limits of the quantum world.

In principle, SS and MFSS could be prepared in the

* alain.sarlette@ugent.be optical domain by letting a coherent laser pulse propagate in a nonlinear medium, whose index of refraction depends on the light pulse intensity [7]. In a Kerr medium, this dependence is linear and the field would evolve from initial coherent state $|\alpha\rangle$ under the action of the Kerr Hamiltonian:

$$
\mathbf{H}_{K}=\zeta_{K} \mathbf{N}+\gamma_{K} \mathbf{N}^{2}
$$

Here $\mathbf{N}$ is the photon number operator, $\zeta_{K}$ measures the linear part of the refraction index and $\gamma_{K}$ is the Kerr frequency describing the strength of the nonlinearity. In the following, we use units such that $\hbar=1$. Note that the collisional interaction Hamiltonian for an $\mathbf{N}$-atom sample in a tightly confining potential or in an optical lattice is similar to $\mathbf{H}_{K}[8]$.

Depending on the interaction time $t_{K}$, the Kerrpropagated state $e^{-i t_{K} \mathbf{H}_{K}}|\alpha\rangle$ can take a number of nonclassical forms [4, Section 7.2], including:

(i) squeezed states for $t_{K} \gamma_{K} \ll \pi$;

(ii) states with 'banana'-shaped Wigner function for slightly larger $t_{K} \gamma_{K}$;

(iii) mesoscopic field state superpositions $\left|k_{\alpha}\right\rangle$ with $k$ equally spaced components for $t_{K} \gamma_{K}=\pi / k[9]$.

(iv) in particular, for $t_{K} \gamma_{K}=\frac{\pi}{2}$, a MFSS of two coherent states with opposite amplitudes:

$$
\left|c_{\tilde{\alpha}}\right\rangle=(|\tilde{\alpha}\rangle+i|-\tilde{\alpha}\rangle) / \sqrt{2},
$$

with $\tilde{\alpha}=\alpha e^{-i \zeta_{K} t_{K}}$.

The top panels on Figure 1 present the Wigner functions of the states (i)-(iv) for a mean photon number $|\alpha|^{2}=$ 2.7 . 


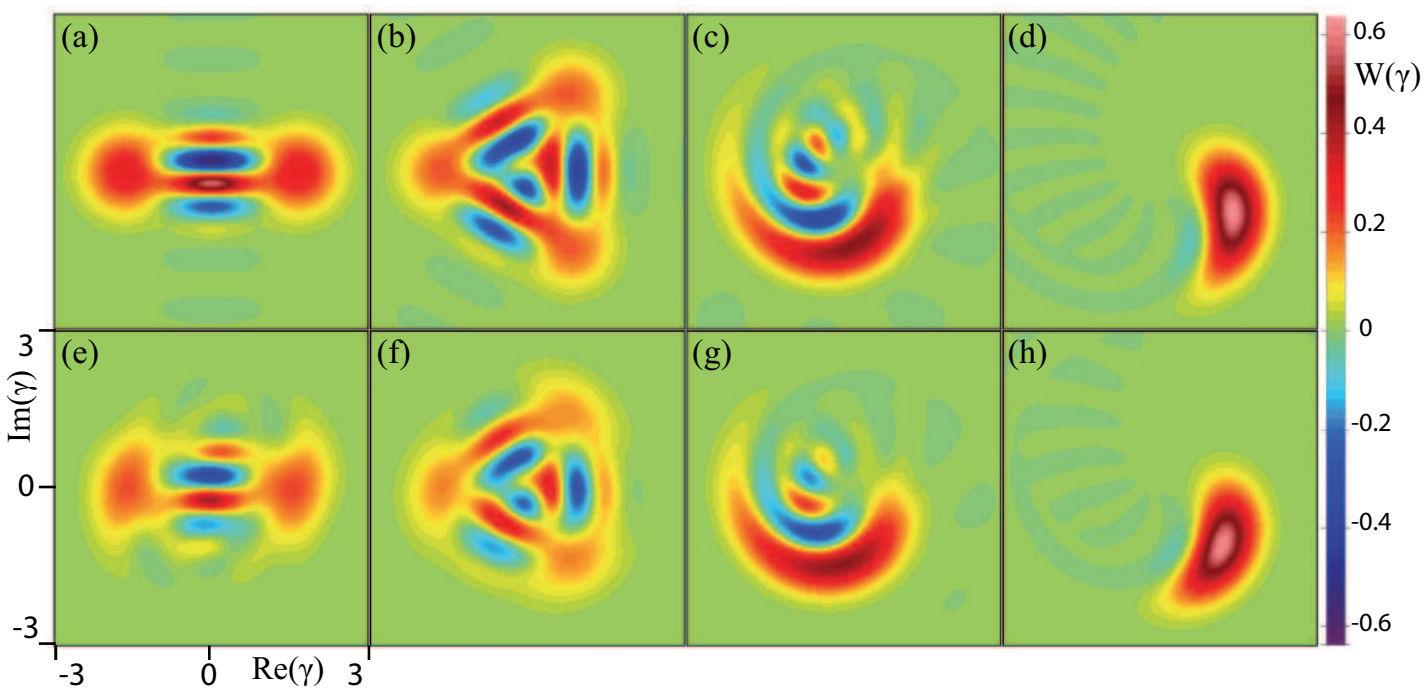

FIG. 1. (Color) Wigner functions $W(\gamma)$, with the same vertical, horizontal axes and color scales for all frames. (a-d) Nonclassical field states $e^{-i t_{K} \mathbf{H}_{K}}|\alpha\rangle$ generated by propagation of an initial coherent state through a Kerr medium, (a) 2-component MFSS given by Eq. (2) for $t_{K} \gamma_{K}=\pi / 2$; (b) 3 -component MFSS for $t_{K} \gamma_{K}=\pi / 3$; (c) 'banana'-state, for $t_{K} \gamma_{K}=0.28$; and (d) squeezed state, for $t_{K} \gamma_{K}=0.08 \ll \pi$. (e-h): similar states stabilized, despite decoherence, by the atomic reservoir onto which we focus in this paper. Frame (e) corresponds to our reference two-component MFSS.

This preparation method by a deterministic unitary evolution is simple in its principle, but its implementation is extraordinarily difficult for propagating light fields, due to the weakness of the Kerr nonlinearity [10].

Other methods for the production of these nonclassical states have been proposed or realized in the context of trapped ions [11, 12] or Cavity Quantum Electrodynamics (CQED) [4, 13-19]. Both systems implement the 'spin-spring' model, the simplest nontrivial quantum situation of a two-level system coupled to a harmonic oscillator, embodied by the harmonic motion of the ion or by a single field mode. Detection-conditioned schemes expand the possibilities of non-classical state production methods, by applying a measurement operation after deterministic production of an intermediate state. Measurement back-action generates different final states conditioned by the stochastic detection outcome [20]. In the microwave CQED context, detection-conditioned preparation of MFSS and ESMS can be achieved by the dispersive interaction of an initial coherent field state with a two-level atom. This atom is initially prepared in a state superposition, and finally detected in an appropriate basis $[4,21]$.

All these preparation techniques do not solve, however, the problem of stabilizing ("protecting") a selected nonclassical state for long times in spite of the unavoidable coupling of the system $\mathcal{S}$ to its environment $\mathcal{E}$. Reservoir engineering can be used to stabilize target quantum states by strongly coupling $\mathcal{S}$ to an "engineered" environment, or reservoir $\mathcal{R}$, a large quantum system with many degrees of freedom. The reservoir is designed so that it drives $\mathcal{S}$, whatever its initial state, towards a unique target 'pointer state', a stable state of $\mathcal{S}$ cou- pled to $\mathcal{R}[22,23]$. The state of $\mathcal{S}$ remains close to this pointer state even in the presence of $\mathcal{E}$, provided $\mathcal{S}$ is more strongly coupled to $\mathcal{R}$ than to $\mathcal{E}$. An engineered reservoir thus achieves much more than the preparation of a target state. It effectively stabilizes the system close to it for arbitrarily long times.

Reservoir engineering is experimentally challenging. Reservoirs made up of lasers and magnetic fields for trapped-ion oscillators have been proposed [19, 24, 25] and demonstrated [26]. Recently, a reservoir has been used to generate entanglement of spin states of macroscopic atomic ensembles [27].

In the context of CQED, the reservoir may be a stream of atoms interacting with the field. An early proposal [28] relied on the so-called 'trapping state conditions' for the micromaser [29], which require a very fine tuning of the parameters and can only be properly achieved in the case of a zero-temperature environment. Reservoirs composed of atoms in combination with external fields have also been proposed to stabilize one-mode squeezed states [30] and two-mode squeezed vacuum entanglement [31].

In [32], we have proposed a robust reservoir engineering method for CQED. It generates and stabilizes nonclassical states of a single mode of the radiation field, including SS and MFSS. The reservoir is made up of a stream of 2-level atoms, each prepared in a coherent superposition of its basis states. They interact one after the other with the field according to the Jaynes-Cummings model before being discarded. The discarding procedure is reminiscent of the "reset" operation performed in other contexts $[26,33]$. The key idea is to let each atom undergo a composite interaction with the field: dispersive, then resonant, then dispersive again. The composite in- 
teraction can be tailored to stabilize a unique pointer state of the form $e^{-i t_{K} \mathbf{H}_{K}}|\alpha\rangle$ for any chosen $t_{K} \gamma_{K}$ and $\alpha$ (at least in principle). It thus gives access to all the non-classical states resulting from the action of a Kerr Hamiltonian upon an initially coherent state.

The method is quite general and could be implemented in a variety of CQED settings, particularly in the active context of circuit QED [34] or in that of microwave CQED, with circular Rydberg atoms and superconducting Fabry-Perot cavities. For the sake of definiteness, we shall focus in this paper on the microwave CQED case, and particularly on the current ENS CQED experiment whose scheme is depicted on Fig. 2. The bottom panels of Fig. 1 present the results of numerical simulations of our reservoir for the ENS experiment, with interaction parameters chosen to reproduce the states generated by the Kerr Hamiltonian (top panels).

The present paper is intended to provide an in-depth description of this single-mode reservoir engineering procedure, with a detailed analysis of the physical mechanism of state stabilization. We also discuss the competition between the engineered reservoir and the ordinary cavity environment, giving simple insights into the finite final fidelity of the prepared state.

We finally extend the scheme proposed in [32] to the stabilization of entangled superpositions of mesoscopic states of two field modes. The atoms of the reservoir undergo a composite interaction with two modes of the same cavity, combining dispersive and resonant parts for each mode. This proposal opens interesting perspectives for studying the interplay between entanglement, nonlocality and decoherence in the context of mesoscopic quantum states.

The paper is organized as follows. We consider the single-mode case for most of the paper and extend it to two modes in the last Section. Section II describes the experimental scheme and the principle of the method. Section III discusses, as a building block for the next Sections, how a stream of atoms in resonant interaction with one field mode stabilizes an approximately coherent field state. Section IV introduces the composite interaction: non-resonant, resonant and non-resonant again. Here, we treat the non-resonant interactions in the 'dispersive regime', assuming a large atom-cavity detuning. We thus introduce in the simplest way the mechanism generating non-classical states. Section V details the more realistic case of intermediate atom-cavity detuning. We show that the main features of Section IV are recovered, exhibiting the robustness of the method. Section VI analyzes the effects of decoherence due to the cavity damping and of imprecisions in the experimental parameters. We find that the method is robust against realistically large imperfections. Section VII finally presents the stabilization of a two-mode ESMS.

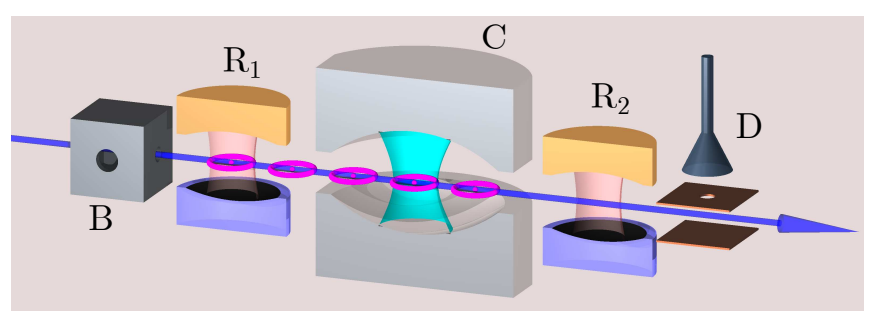

FIG. 2. (Color online) Scheme of the current ENS CQED experiment.

\section{GENERAL DESCRIPTION}

The scheme of the ENS experiment is depicted on Fig. 2 (see $[4,15]$ for details). A microwave field of frequency $\omega_{c}$ is trapped in the superconducting cavity $C$ (damping time $T_{c}=65 \mathrm{~ms}$ ). Atoms are sent one after the other through $C$. They cross its standing wave Gaussian mode at a constant, adjustable velocity $v$. The mode interacts with the transition between the two atomic levels $|g\rangle$ and $|e\rangle$ (circular Rydberg states with principal quantum numbers 50 and 51). A static electric field applied across the cavity mirrors is used to adjust the atomic transition frequency $\omega_{0}$ and hence the atom-cavity detuning $\delta=\omega_{0}-\omega_{c} \ll \omega_{c}$ via the Stark effect. Varying the electric field during the atom-field interaction makes it possible to engineer the detuning profile $\delta(t)$. Zero and small $\delta$ values are used for the resonant and non-resonant parts of the interaction. Making $\delta$ very large allows to effectively turn off the atom-field interaction.

We describe the atom and field states in a frame rotating at frequency $\omega_{c}$, such that the field state remains constant in absence of interaction. The atoms are prepared in state $|g\rangle$ in $B$, by a time-resolved laser and radio-frequency excitation of a velocity-selected thermal rubidium atomic beam. Before entering $C$, the atoms are cast in a coherent superposition of $|g\rangle$ and $|e\rangle$ in the low-quality cavity $R_{1}$ ("first Ramsey zone"), driven by a classical microwave source at frequency $\omega_{c}$. We set the phase of the coherent superposition for each atom so that it enters the cavity in the initial state $\left|u_{\mathrm{at}}\right\rangle=\cos (u / 2)|g\rangle+\sin (u / 2)|e\rangle$ with $u>0$. In a Bloch sphere representation with $|e\rangle$ at the north pole, $\left|u_{\text {at }}\right\rangle$ corresponds to a vector in the Z-X plane at an angle $u$ with the north-to-south vertical axis.

A second classical microwave pulse in the second Ramsey zone $R_{2}$ is followed by a detection in the $\{|e\rangle,|g\rangle\}$ basis in the field-ionization detector $D$. This detection scheme amounts to a projective measurement of the atomic state at the exit of $C$, in a basis that can be chosen by properly setting the microwave pulse in $R_{2}$. For the engineered reservoir operation, the result of this final atomic state detection is irrelevant. Detection results are however useful in other phases of the experiment. In particular, they may be used to reconstruct the field state generated by the engineered reservoir, using a method described in [15]. 
Let us first consider the atom-cavity interaction for one atom that crosses cavity axis at $t=0$. It is ruled by the Jaynes-Cummings Hamiltonian $\mathbf{H}_{J C}$. Neglecting far offresonant terms (rotating wave approximation, negligible interaction with other cavity modes), we have:

$\mathbf{H}_{J C}=\frac{\delta(t)}{2}(|e\rangle\langle e|-| g\rangle\langle g|)+i \frac{\Omega(s)}{2}\left(|g\rangle\left\langle e\left|\mathbf{a}^{\dagger}-\right| e\right\rangle\langle g| \mathbf{a}\right)$,

where $\Omega(s)$ is the atom-cavity coupling strength (vacuum Rabi frequency) at position $s=v t$ along the atomic trajectory; $\mathbf{a}$ is the photon annihilation operator in $C$. The photon number operator $\mathbf{N}=\mathbf{a}^{\dagger} \mathbf{a}=\sum_{n} n|n\rangle\langle n|$ defines the Fock states basis $\{|n\rangle\}$.

The coupling strength $\Omega(s)$ is determined by the atomic transition parameters and by the cavity mode geometry. It writes here $\Omega(s)=\Omega_{0} e^{-s^{2} / w^{2}}$, with $\Omega_{0} / 2 \pi=50 \mathrm{kHz}$ and $w=6 \mathrm{~mm}$. To get a finite total interaction duration $T$, we assume that the coupling cancels when $|s|>1.5 \mathrm{w}$. The total interaction time of the atom with the field is thus $T=3 w / v$. We have checked in numerical simulations that this truncation of interaction time has negligible effects.

The evolution operator, or propagator $\mathbf{U}$ associated to $\mathbf{H}_{J C}$, expresses the transformation that the joint atom-cavity state undergoes during interaction. The Schrödinger equation for $\mathbf{U}$, starting at the initial time $t=t_{0}$, is:

$$
\frac{d}{d t} \mathbf{U}(t)=-i \mathbf{H}_{J C}(t) \mathbf{U}(t) \text { with } \mathbf{U}\left(t_{0}\right)=\mathbf{I},
$$

where $\mathbf{I}$ is the identity operator. We call $\mathbf{U}_{T}$ the propagator obtained by integration of Eq. (4) over one full atom-cavity interaction, that lasts from $-T / 2$ to $T / 2$.

We represent the action of $\mathbf{U}_{T}$ over the field state by the operators $\mathbf{M}_{g}^{\mathbf{U}_{T}}$ and $\mathbf{M}_{e}^{\mathbf{U}_{T}}$ such that:

$$
\mathbf{U}_{T}\left(\left|u_{\mathrm{at}}\right\rangle|\psi\rangle\right)=|g\rangle \mathbf{M}_{g}^{\mathbf{U}_{T}}|\psi\rangle+|e\rangle \mathbf{M}_{e}^{\mathbf{U}_{T}}|\psi\rangle,
$$

for any pure initial field state $|\psi\rangle$. Tracing over the final atomic state, the modification of the field density operator $\rho$ due to the interaction with the atom is thus finally given by the Kraus map [35]

$$
\rho \rightarrow \mathbf{M}_{g}^{\mathbf{U}_{T}} \rho \mathbf{M}_{g}^{\mathbf{U}_{T} \dagger}+\mathbf{M}_{e}^{\mathbf{U}_{T}} \rho \mathbf{M}_{e}^{\mathbf{U}_{T}^{\dagger}} .
$$

To implement reservoir action, we let a stream of atoms interact with the field one after the other, each with the same parameter set (detuning profile, atom velocity $v$ and initial state $\left.\left|u_{\text {at }}\right\rangle=\cos (u / 2)|g\rangle+\sin (u / 2)|e\rangle\right)$. Thus each atom affects the field according to Eq. (5). The interaction with atom $k+1$ begins as soon as the interaction with atom $k$ ends. Consecutive atoms are thus separated by the time interval $T$. We call $\rho_{k}$ the cavity state just after the interaction with the $k$ th atom obtained by tracing over this atom's irrelevant final state. This operator $\rho_{k}$ evolves as:

$$
\rho_{k}=\mathbf{M}_{g}^{\mathbf{U}_{T}} \rho_{k-1} \mathbf{M}_{g}^{\mathbf{U}_{T}^{\dagger} \dagger}+\mathbf{M}_{e}^{\mathbf{U}_{T}} \rho_{k-1} \mathbf{M}_{e}^{\mathbf{U}_{T}^{\dagger}} .
$$
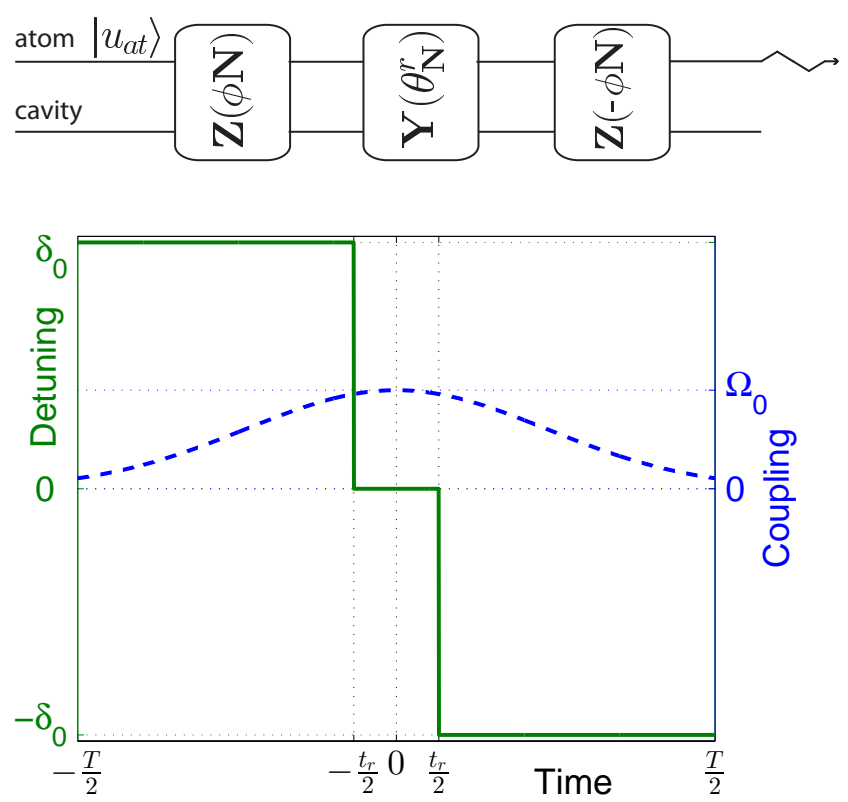

FIG. 3. (Color online) Top frame: scheme of the propagators corresponding to the successive steps in the composite interaction. Bottom frame: schematic time profile of $\delta(t)$ (difference between the tunable frequency $\omega_{0}$ of the atomic transition and the fixed frequency $\omega_{c}$ of the single cavity mode - full line) and $\Omega(v t)$ (dashed line) during cavity crossing by one atomic sample. We take $t=0$ when the atom is at cavity center.

State stabilization by reservoir engineering amounts to tailoring a Kraus map for the field from a constrained physical setting. Our goal is to stabilize a pure pointer state $\rho_{\infty}=\left|\psi_{\infty}\right\rangle\left\langle\psi_{\infty}\right|$. It is then necessary to build the Kraus map such that it admits $\rho_{\infty}$ as a fixed point. Starting with $\rho_{k-1}=\rho_{\infty}$, the right-hand side of Eq. (6) is a statistical mixture of two pure states. Those two pure states must be both equal to $\left|\psi_{\infty}\right\rangle$ if their mixture is the pure state $\rho_{k}=\rho_{\infty}$. Thus, $\left|\psi_{\infty}\right\rangle$ must be an eigenstate of both $\mathbf{M}_{g}^{\mathbf{U}_{T}}$ and $\mathbf{M}_{e}^{\mathbf{U}_{T}}$.

We have shown in [32] that it is possible to engineer the atom-cavity interaction so that the Kraus map leaves invariant $\left|\psi_{\infty}\right\rangle \approx e^{-i t_{K} \mathbf{H}_{K}}|\alpha\rangle$, in which $\alpha$ and $\gamma_{K} t_{K}$ in Eq.(1) can be chosen at will. Explicitly, we build $\mathbf{U}_{T}$ by sandwiching a resonant interaction $(\delta=0$ for $\left.t \in\left[-t_{r} / 2, t_{r} / 2\right]\right)$ symmetrically between two dispersive interactions with opposite detuning: $\delta=\delta_{0}$ before the resonant interaction, $\delta=-\delta_{0}$ after. (We assume a positive $\delta_{0}$ value for the sake of definiteness.)

This experimental sequence is illustrated on Fig. 3. Each resonant or dispersive part of the interaction is characterized by a set of parameters that we note $q=$ $\left(t_{1}, t_{2}, v, \delta\right)$ where $t_{1}$ is the start time, $t_{2}$ is the stop time and $\delta$ is the chosen (constant) detuning. The corresponding propagators are denoted $\mathbf{U}_{q}$.

To compute these propagators, we use the fact that each two-dimensional subspace spanned by $(\mid g, n+$ $1\rangle,|e, n\rangle)$ is invariant under the action of $\mathbf{H}_{J C}$. The 
state $|g, 0\rangle$ does not take part in the evolution. We can thus view $\mathbf{U}_{q}$ as applying rotations in parallel on a set of Bloch spheres $B_{n}(n=0,1, \ldots)$, whose respective $Z$-axes are defined by $|g, n+1\rangle$ at the south-pole and $|e, n\rangle$ at the north-pole. These rotations can be decomposed into rotations around the $X-, Y$ - and $Z$-axes of the Bloch spheres. We use the notation $f_{\mathbf{N}}=f(\mathbf{N})=\sum_{n} f(n)|n\rangle\langle n|=$ $\sum_{n} f_{n}|n\rangle\langle n|$ for arbitrary functions $f$ of $n$ and we use the relation:

$$
\mathbf{a} f(\mathbf{N})=f(\mathbf{N}+\mathbf{I}) \mathbf{a} \text {. }
$$

We can then define generalized rotations, acting in parallel around the associated axes of all Bloch spheres with photon-number-dependent rotation angle:

$$
\begin{aligned}
\mathbf{X}\left(f_{\mathbf{N}}\right)= & |g\rangle\left\langle g\left|\cos \left(f_{\mathbf{N}} / 2\right)+\right| e\right\rangle\langle e| \cos \left(f_{\mathbf{N}+\mathbf{I}} / 2\right) \\
& -i|e\rangle\left\langle g\left|\mathbf{a} \frac{\sin \left(f_{\mathbf{N}} / 2\right)}{\sqrt{\mathbf{N}}}-i\right| g\right\rangle\langle e| \frac{\sin \left(f_{\mathbf{N}} / 2\right)}{\sqrt{\mathbf{N}}} \mathbf{a}^{\dagger}, \\
\mathbf{Y}\left(f_{\mathbf{N}}\right)= & |g\rangle\left\langle g\left|\cos \left(f_{\mathbf{N}} / 2\right)+\right| e\right\rangle\langle e| \cos \left(f_{\mathbf{N}+\mathbf{I}} / 2\right) \\
& -|e\rangle\left\langle g\left|\mathbf{a} \frac{\sin \left(f_{\mathbf{N}} / 2\right)}{\sqrt{\mathbf{N}}}+\right| g\right\rangle\langle e| \frac{\sin \left(f_{\mathbf{N}} / 2\right)}{\sqrt{\mathbf{N}}} \mathbf{a}^{\dagger} \\
\mathbf{Z}\left(f_{\mathbf{N}}\right)= & |g\rangle\left\langle g\left|e^{i f_{\mathbf{N}} / 2}+\right| e\right\rangle\langle e| e^{-i f_{\mathbf{N}+\mathbf{I}} / 2}
\end{aligned}
$$

As shown in Sections III and IV, $\mathbf{Y}\left(f_{\mathbf{N}}\right)$ with $f(n)$ proportional to $\sqrt{n+1}$ corresponds to a resonant interaction and $\mathbf{Z}\left(f_{\mathbf{N}}\right)$ with $f(n)$ proportional to $n$ corresponds to a non-resonant interaction in the dispersive regime $\delta \gg \Omega$. See also Appendix A for more details.

\section{ENGINEERED RESERVOIR FOR COHERENT STATE STABILIZATION}

The coherent state $|\alpha\rangle$ is obtained by the action of the displacement operator $\mathbf{D}_{\alpha}=e^{\alpha \mathbf{a}^{\dagger}-\alpha^{\dagger} \mathbf{a}}$ onto the vacuum [4]:

$$
|\alpha\rangle=\mathbf{D}_{\alpha}|0\rangle=e^{-|\alpha|^{2} / 2} \sum_{n} \frac{\alpha^{n}}{\sqrt{n !}}|n\rangle .
$$

We show here how a brief resonant interaction $(\delta=0)$ with weakly excited atoms provides an engineered reservoir whose pointer state is close to a coherent state [32].

Stabilization of coherent states is not an amazing feat. They are indeed directly generated by a classical radiation source weakly coupled to the cavity. This is a routine operation in microwave CQED experiments. However, the situation described in this Section is an essential building block for the stabilization of nonclassical states. Moreover, it is an interesting micromaser situation $[28,36]$, in which the small excitation of the atoms leads to a steady state with finite energy even though the cavity is assumed to be lossless.

We consider a resonant interaction over a time interval $t_{r}$, corresponding to the parameter set $r=$ $\left(-t_{r} / 2, t_{r} / 2, v, 0\right)$. A direct integration yields the associated propagator:

$$
\begin{aligned}
\mathbf{U}_{r}= & \mathbf{Y}\left(\theta_{\mathbf{N}}^{r}\right) \\
= & |g\rangle\left\langle g\left|\cos \left(\theta_{\mathbf{N}}^{r} / 2\right)+\right| e\right\rangle\langle e| \cos \left(\theta_{\mathbf{N}+\mathbf{I}}^{r} / 2\right) \\
& -|e\rangle\left\langle g\left|\mathbf{a} \frac{\sin \left(\theta_{\mathbf{N}}^{r} / 2\right)}{\sqrt{\mathbf{N}}}+\right| g\right\rangle\langle e| \frac{\sin \left(\theta_{\mathbf{N}}^{r} / 2\right)}{\sqrt{\mathbf{N}}} \mathbf{a}^{\dagger},
\end{aligned}
$$

with

$$
\theta_{n}^{r}=\theta_{r} \sqrt{n}, \quad \theta_{r}=\int_{-t_{r} / 2}^{t_{r} / 2} \Omega(v t) d t .
$$

This readily yields:

$$
\begin{aligned}
& \mathbf{M}_{g}^{\mathbf{U}_{r}}=\cos \left(\frac{u}{2}\right) \cos \left(\theta_{\mathbf{N}}^{r} / 2\right)+\sin \left(\frac{u}{2}\right) \frac{\sin \left(\theta_{\mathbf{N}}^{r} / 2\right)}{\sqrt{\mathbf{N}}} \mathbf{a}^{\dagger} \\
& \mathbf{M}_{e}^{\mathbf{U}_{r}}=\sin \left(\frac{u}{2}\right) \cos \left(\theta_{\mathbf{N}+\mathbf{I}}^{r} / 2\right)-\cos \left(\frac{u}{2}\right) \mathbf{a} \frac{\sin \left(\theta_{\mathbf{N}}^{r} / 2\right)}{\sqrt{\mathbf{N}}} .
\end{aligned}
$$

A pointer state $\left|\psi_{\infty}\right\rangle$ of the reservoir associated to this resonant interaction must be an eigenstate of both $\mathbf{M}_{g}$ and $\mathbf{M}_{e}$ given by Eq.(14). Writing $\left|\psi_{\infty}\right\rangle=\sum \psi_{n}|n\rangle$ in the Fock states basis, we get a condition on the coefficients $\psi_{n}$, for $n=0,1,2, \ldots$ :

$$
\sin \left(\theta_{n+1}^{r} / 2\right) \psi_{n+1}=\tan \frac{u}{2}\left(1+\cos \left(\theta_{n+1}^{r} / 2\right)\right) \psi_{n} .
$$

This relation allows us to compute all the $\psi_{n}$ coefficients starting from $\psi_{0} \neq 0$, except if $\sin \left(\theta_{m}^{r} / 2\right)=0$ for some $m$. This case corresponds to the existence of a trapping state $|m-1\rangle$ [37], which is then uncoupled from $|m\rangle$ in the reservoir action. The Hilbert subspace spanned by the photon numbers $\leq(m-1)$ is then decoupled from its orthogonal complement, spanned by photon numbers $\geq m$, such that the steady state depends on the initial conditions. Since all states considered in the remainder of the paper have an energy lower than 20 photons, we truncate the Hilbert space to $n \leq n_{\max }=50$. This allows us to avoid trapping states by restricting $\theta_{r}$ to small enough values such that $\sin \left(\theta_{n+1}^{r} / 2\right) \neq 0$ for all $0 \leq n \leq n_{\max }$. Dividing (15) by $\sin \left(\theta_{n+1}^{r} / 2\right)$ gives then the recurrence relation:

$$
\psi_{n+1}=\frac{\tan (u / 2)}{\tan \left(\theta_{n+1}^{r} / 4\right)} \psi_{n},
$$

which defines a unique normalized pointer state.

For $\left(\theta_{n_{\max }}^{r} / 4\right)^{2} \ll 1$, Eq.(16) can be approximated by $\psi_{n+1} \approx \frac{4 \tan (u / 2)}{\theta_{r} \sqrt{n+1}} \psi_{n}$, which defines a coherent state $\left|\alpha_{\infty}\right\rangle$ with $\alpha_{\infty}=\frac{4 \tan (u / 2)}{\theta_{r}}$ (compare with the last member of Eq. (11)).

This $\alpha_{\infty}$ value can be retrieved by a simplified physical reasoning, as in [32]. Assume that the cavity already contains a large coherent field of amplitude $\alpha \gg 1$. The incoming atoms then undergo a resonant Rabi rotation in this field. The atomic Bloch vector starts initially towards the south pole of the Bloch sphere. It rotates by an angle $-\theta_{r} \alpha$. If $\theta_{r} \alpha<2 u$ (resp. $\theta_{r} \alpha>2 u$ ), the final 
atomic state has a lower (resp. larger) energy than the initial one, i.e. in average gives energy to (resp. draws energy from) the field. The energy exchange thus stabilizes a field with the amplitude $\alpha_{\infty}=2 u / \theta_{r}$.

We have numerically examined the fidelity $F=$ $\left|\left\langle\psi_{\infty} \mid \alpha_{*}\right\rangle\right|^{2}$ of the pointer state $\left|\psi_{\infty}\right\rangle$ defined by Eq. (16) with respect to a coherent state $\left|\alpha_{*}\right\rangle$ of the same mean photon number $\left|\alpha_{*}\right|^{2}=\left\langle\psi_{\infty}|\mathbf{N}| \psi_{\infty}\right\rangle$. Figure 4(a) represents this mean photon number in grayscale as a function of $u$ and $\theta_{r}$. We limit the plot to $\theta_{r}<$ $(2 \pi) / \sqrt{n_{\max }} \approx 0.88$ to avoid trapping states, and to $\theta_{r}>5 \tan (u / 2) / \sqrt{n_{\max }}$ to remain in the truncated Hilbert space (top left corner cut off). The coherent state approximation for $\left|\psi_{\infty}\right\rangle$ remarkably holds for a range of $u$ and $\theta_{r}$ much wider than that predictable from the qualitative discussion above. We find a fidelity $F$ larger than $99 \%$ over the whole shaded area in figure $4(\mathrm{a})$.

Convergence towards $\left|\alpha_{\infty}\right\rangle$ can be simply analyzed in the limit of small $u, \theta_{r}$. Expansion of Eq. (14) to second order in $u$ and $\theta_{\mathbf{N}}^{r}$ yields the Kraus map:

$$
\begin{aligned}
\rho_{k+1} \approx & \rho_{k}+\frac{u \theta_{r}}{4}\left(\left[\mathbf{a}^{\dagger}, \rho_{k}\right]-\left[\mathbf{a}, \rho_{k}\right]\right) \\
& -\frac{\theta_{r}^{2}}{8}\left(\mathbf{N} \rho_{k}+\rho_{k} \mathbf{N}-2 \mathbf{a} \rho_{k} \mathbf{a}^{\dagger}\right) .
\end{aligned}
$$

We define $\tilde{\rho}=\mathbf{D}_{-\alpha_{\infty}} \rho \mathbf{D}_{\alpha_{\infty}}$, such that $\rho=\left|\alpha_{\infty}\right\rangle\left\langle\alpha_{\infty}\right|$ corresponds to $\tilde{\rho}=|0\rangle\langle 0|$. A few calculations show that Eq. (17) is equivalent to

$$
\tilde{\rho}_{k+1}=\tilde{\rho}_{k}-\frac{\theta_{r}^{2}}{8}\left(\mathbf{N} \tilde{\rho}_{k}+\tilde{\rho}_{k} \mathbf{N}-2 \mathbf{a} \tilde{\rho}_{k} \mathbf{a}^{\dagger}\right) .
$$

This is a finite difference version of the standard Lindblad equation describing the damping of a harmonic oscillator coupled to a zero temperature bath. It drives any initial state towards the vacuum $\tilde{\rho}=|0\rangle\langle 0|$, with a relaxation rate proportional to $\theta_{r}^{2}$. This analogy shows that the initial Kraus map [Eq. (17)] drives any initial cavity state towards the coherent state $\rho=\left|\alpha_{\infty}\right\rangle\left\langle\alpha_{\infty}\right|$, with $\alpha_{\infty}=2 u / \theta_{r}$. A smaller $\theta_{r}$ value, i.e. a shorter interaction time of each atom with the field, leads to a higher energy pointer state (for a given $u$ ), but to a lower convergence rate (independent of $u$ ).

An alternative line of reasoning directly considers Eq. (17), but assumes that the field always remains in a coherent state. In a second-order approximation of Eq. (17) in $u, \theta_{r}$ [38], the amplitude $\alpha_{k}$ of this coherent state evolves as:

$$
\alpha_{k+1}=\left(1-\theta_{r}^{2} / 8\right) \alpha_{k}+u \theta_{r} / 4 .
$$

This first-order system has the explicit solution $\alpha_{k}=$ $\left(1-\theta_{r}^{2} / 8\right)^{k}\left(\alpha_{0}-\alpha_{\infty}\right)+\alpha_{\infty}$ starting from $\alpha_{0}$ at $k=0$. Noting that $\log \left|\left\langle\alpha_{\infty} \mid \alpha_{k}\right\rangle\right|^{2}=-\left|\alpha_{k}-\alpha_{\infty}\right|^{2}$, the fidelity indicator $\left.\log |\log |\left\langle\alpha_{\infty} \mid \alpha_{k}\right\rangle\right|^{2}|=\log | \alpha_{0}-\left.\alpha_{\infty}\right|^{2}-\lambda_{\text {conv }} k$ decreases linearly in $k$ towards $-\infty$, with a slope $\lambda_{\text {conv }}=$ $2\left|\log \left(1-\theta_{r}^{2} / 8\right)\right|$. This slope measures the exponential convergence rate of $\left|\alpha_{k}-\alpha_{\infty}\right|^{2}$, which increases with $\theta_{r}$ and is independent of $u$.
Numerical simulations of Eq. (6) with the exact Kraus map [Eq. (14)] vindicate this approximate analysis. Figure 4(b) shows the evolution of $\log \left|\log \left\langle\psi_{\infty}\left|\rho_{k}\right| \psi_{\infty}\right\rangle\right|$ as a function of the number of atom-field interactions $k$, starting from the vacuum $\rho_{0}=|0\rangle\langle 0|$, with the real Kraus map associated to $\mathbf{U}_{r}$. The evolution is linear, as predicted by the simplified model. We have checked that this linearity holds for a large range of parameter values. This allows us to characterize convergence speed by the slope $\lambda_{\text {conv }}$ of that approximate evolution. Fig. 4(c) shows the dependence of $\lambda_{\text {conv }}$ in $\theta_{r}$, for two different $u$ values: $u=0.1$ (dotted curve) and $u=1$ (dashed curve, which does not extend to low $\theta_{r}$ values, according to the accessible domain on Fig. 4(a)). They closely follow the simplified model (full line), which is independent of $u$ and slightly overestimates convergence speed.

\section{KERR HAMILTONIAN SIMULATION IN THE DISPERSIVE REGIME}

We now discuss the case of a full composite interaction, with the detuning profile $\delta(t)$ represented on Fig. 3. The full propagator

$$
\mathbf{U}_{T}=\mathbf{U}_{d_{2}} \mathbf{U}_{r} \mathbf{U}_{d_{1}}
$$

is the concatenation of three unitary operators corresponding first to a non-resonant interaction with parameters $d_{1}=\left(-T / 2,-t_{r} / 2, v, \delta_{0}\right)$ as the atom enters the cavity; then to a resonant interaction around cavity center with $q=r$ (see previous Section); and finally to a second non-resonant interaction with $d_{2}=\left(t_{r} / 2, T / 2, v,-\delta_{0}\right)$ as the atom leaves the cavity. The exact expression of $\mathbf{U}_{r}$ is given by Eq. (12). The propagators $\mathbf{U}_{d_{1}}$ and $\mathbf{U}_{d_{2}}$ are computed in Appendix A, assuming that $\delta_{0}$ and $v$ satisfy the adiabatic approximation condition [Eq. (A1)].

To get a simple insight into the physics of nonclassical states stabilization, the present Section focuses (like [32]) on the simple case in which the two non-resonant interactions take place in the dispersive regime, i.e. $\delta_{0} \gg \Omega_{0}$. This avoids considering spurious population transfers during the non-resonant interactions, as atomic levels dressed by the cavity field almost coincide with the bare levels $|e\rangle$ and $|g\rangle$. The dispersive propagators, deduced from Eq. (A7) in Appendix A, then write:

$$
\mathbf{U}_{d_{1}} \approx \mathbf{U}_{d_{2}}^{\dagger} \approx \mathbf{Z}\left(\phi_{\mathbf{N}}^{d}\right)
$$

with $\phi_{\mathbf{N}}^{d}=\phi_{\gamma} \mathbf{N}+\phi_{\zeta}$,

where $\phi_{\gamma}=1 /\left(2 \delta_{0}\right) \int_{-T / 2}^{-t_{r} / 2} \Omega^{2}(v t) d t$ is a phase shift per photon and $\phi_{\zeta}=\delta_{0}\left(T-t_{r}\right) / 2$ reflects the free atom evolution in the interaction representation at cavity frequency. 

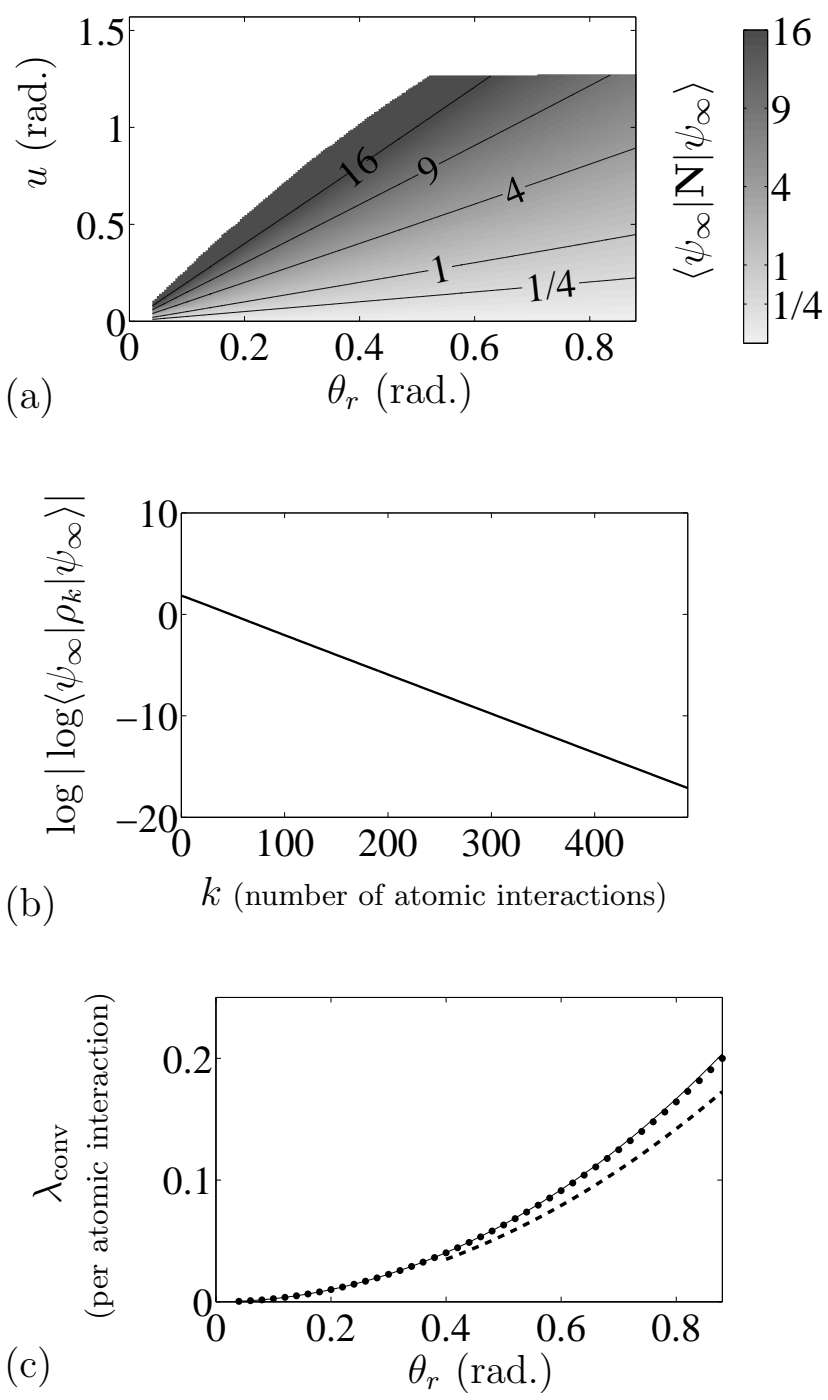

FIG. 4. Reservoir action with resonant atom-field interaction. (a) Mean photon number $\left\langle\psi_{\infty}|\mathbf{N}| \psi_{\infty}\right\rangle$ of the pointer state $\left|\psi_{\infty}\right\rangle$. Grayscale axis is linear in $\sqrt{\left\langle\psi_{\infty}|\mathbf{N}| \psi_{\infty}\right\rangle}$. The shaded zone is delimited such that the corresponding states have at least a $99 \%$ fidelity $\left|\left\langle\psi_{\infty} \mid \alpha_{*}\right\rangle\right|^{2}$ to a coherent state $\left|\alpha_{*}\right\rangle$ of same mean photon number $\left|\alpha_{*}\right|^{2}=\left\langle\psi_{\infty}|\mathbf{N}| \psi_{\infty}\right\rangle$. On the top left corner, pointer states have significant population outside the truncated Hilbert space. On the top right part, $\left|\left\langle\psi_{\infty} \mid \alpha_{*}\right\rangle\right|^{2}$ drops to $\sim 98 \%$ as $u$ approaches $\pi / 2$. (b) Evolution of the fidelity indicator $\log \left|\log \left\langle\psi_{\infty}\left|\rho_{k}\right| \psi_{\infty}\right\rangle\right|$ as a function of the number of atom-field interactions (i.e. Kraus map iterations) $k$, starting from vacuum $\rho_{0}=|0\rangle\langle 0|$. For illustration we have chosen an arbitrary typical case with parameters $u=0.5$ and $\theta_{r}=0.4$, giving $\left\langle\psi_{\infty}|\mathbf{N}| \psi_{\infty}\right\rangle=6.21$. (c) Convergence rate $\lambda_{\text {conv }}$ as a function of $\theta_{r}$ for $u=0.1$ (dotted curve) and $u=1$ (dashed curve). Dependency in $u$ is small. We also represent (full curve) the analytic result of the simplified model [Eq. (19)]. This model slightly overestimates the convergence speed.
The full propagator then writes

$$
\begin{aligned}
\mathbf{U}_{T} \approx \mathbf{U}_{d}= & \mathbf{Z}\left(-\phi_{\mathbf{N}}^{d}\right) \mathbf{U}_{r} \mathbf{Z}\left(\phi_{\mathbf{N}}^{d}\right) \\
= & |g\rangle\left\langle g\left|\cos \theta_{\mathbf{N}}^{r} / 2+\right| e\right\rangle\langle e| \cos \theta_{\mathbf{N}+\mathbf{I}}^{r} / 2 \\
& -|e\rangle\langle g| \mathbf{a} \frac{\sin \theta_{\mathbf{N}}^{r} / 2}{\sqrt{\mathbf{N}}} e^{i\left(\phi_{\gamma} \mathbf{N}+\phi_{\zeta}\right)} \\
& +|g\rangle\langle e| \frac{\sin \theta_{\mathbf{N}}^{r} / 2}{\sqrt{\mathbf{N}}} e^{-i\left(\phi_{\gamma} \mathbf{N}+\phi_{\zeta}\right)} \mathbf{a}^{\dagger}
\end{aligned}
$$

where $\theta_{\mathbf{N}}^{r}$ is defined by Eq. (13). The two opposite dispersive interactions have no net effect on the terms in which the atom remains in the same state during the resonant interaction $\mathbf{U}_{r}$. In contrast, for the terms in which the atomic level changes during the resonant interaction, the dispersive phase shifts add up, as $\mathbf{Z}$ does not commute with $\mathbf{U}_{r}$. The global evolution thus associates a phase shift to each term of $\mathbf{U}_{r}$ that changes the field energy. An increasing field energy corresponds to a decrease of the field phase and vice versa. These correlated phase and amplitude shifts suggest that $\mathbf{U}_{d}$ might stabilize a coherent state distorted by amplitude-dependent phase shifts, similar to the result of the propagation through a Kerr medium.

It turns out that the dispersive interaction can indeed be expressed by an operator acting on the field only. Let us define the Hermitian operator $h_{\mathbf{N}}^{d}$ by:

$$
h_{\mathbf{N}}^{d}=\phi_{\gamma}\left(\mathbf{N}^{2}+\mathbf{N}\right) / 2+\phi_{\zeta} \mathbf{N} \text {. }
$$

Thanks to the commutation identity [Eq. (7)] we have $e^{-i h_{\mathbf{N}}^{d}} \mathbf{a} e^{i h_{\mathbf{N}}^{d}}=\mathbf{a} e^{i\left(\phi_{\gamma} \mathbf{N}+\phi_{\zeta}\right)}$ and

$$
\mathbf{U}_{d}=e^{-i h_{\mathbf{N}}^{d}} \mathbf{U}_{r} e^{i h_{\mathbf{N}}^{d}}
$$

Thus, $\mathbf{U}_{d}$ is equivalent to $\mathbf{U}_{r}$ modulo a basis change on field state alone defined by the unitary operator $e^{-i h_{\mathbf{N}}^{d}}$.

In other words, as $\rho$ evolves under the Kraus map associated to $\left(\mathbf{M}_{g}^{\mathbf{U}_{d}}, \mathbf{M}_{e}^{\mathbf{U}_{d}}\right)$, then $\rho^{h}=e^{i h_{\mathbf{N}}^{d}} \rho e^{-i h_{\mathbf{N}}^{d}}$, evolves under the Kraus map associated to $\left(\mathbf{M}_{g}^{\mathbf{U}_{r}}, \mathbf{M}_{e}^{\mathbf{U}_{r}}\right)$. It follows from Section III that $\rho^{h}$ converges towards a coherent pointer state $\left|\alpha_{\infty}\right\rangle$. Therefore, $\rho$ converges with the same convergence rate towards a nonclassical pointer state $\exp \left[-i h_{\mathbf{N}}^{d}\right]\left|\alpha_{\infty}\right\rangle$.

The effective Hamiltonian $h_{\mathbf{N}}^{d} / t_{K}$ is equal to the Kerr Hamiltonian $\mathbf{H}_{K}$, with $\gamma_{K} t_{K}=\phi_{\gamma} / 2$ and $\zeta_{K} t_{K}=$ $\left(\phi_{\zeta}+\phi_{\gamma} / 2\right)$. The engineered reservoir thus stabilizes the nonclassical pointer states $e^{-i t_{K} \mathbf{H}_{K}}\left|\alpha_{\infty}\right\rangle$ which would be produced by propagation through a Kerr medium (see Fig. 1). Tuning of $T$ and $\delta_{0}$ allows us to choose $\phi_{\gamma}$ at will. We can thus prepare and stabilize a whole class of states as described in Section I. In particular, for $\phi_{\gamma}=\pi$, we get the MFSS $\left|c_{\tilde{\alpha}_{\infty}}\right\rangle=\left(\left|\tilde{\alpha}_{\infty}\right\rangle+i\left|-\tilde{\alpha}_{\infty}\right\rangle\right) / \sqrt{2}$ with $\tilde{\alpha}_{\infty}=e^{-i\left(\phi_{\zeta}+\pi / 2\right)} \alpha_{\infty}$. Note that the stabilization of this two-component MFSS is the most demanding, in the sense that it requires the longest dispersive interaction time.

Our analysis so far considers the limit of small $\Omega / \delta_{0}$. Reaching notable $\phi_{\gamma}$ in this case requires a large dispersive interaction time $\left(T-t_{r}\right) / 2$. This requirement can be 
prohibitive for several reasons. First, in the experimental context of Fig. 2, the minimal achievable atomic velocity (a few tens of $\mathrm{m} / \mathrm{s}$ in the ENS setup) limits the maximal accessible values of $T=3 w / v$. Second, a larger $T$ means less frequent atom-field interactions and thus a weaker reservoir, implying a less efficient protection of the target state against decoherence induced by cavity relaxation. The next Section therefore discusses non-resonant interaction with moderate $\Omega / \delta_{0}$ values, which allows us to reach significant dispersive effects within moderate interaction times.

\section{REGIME OF ARBITRARY DETUNING}

For moderate $\Omega / \delta_{0}$ values, we must use a more precise expression of the propagator for the non-resonant interactions (parameter sets $d_{1}$ and $d_{2}$ ), by applying the adiabatic approximation to the actual dressed states (instead of $|g, n+1\rangle$ and $|e, n\rangle$ as in Eq. (21) when assuming $\left.\delta_{0} \gg \Omega_{0}\right)$. Developments detailed in Appendix A lead to:

$$
\mathbf{U}_{T} \approx \mathbf{U}_{c}=\mathbf{Z}\left(-\phi_{\mathbf{N}}\right) \mathbf{X}\left(\xi_{\mathbf{N}}\right) \mathbf{Y}\left(\theta_{\mathbf{N}}^{r}\right) \mathbf{X}\left(\xi_{\mathbf{N}}\right) \mathbf{Z}\left(\phi_{\mathbf{N}}\right),
$$

with

$$
\begin{aligned}
\phi_{n} & =\delta_{0} \int_{-T / 2}^{-t_{r} / 2} \sqrt{1+n\left(\Omega(v t) / \delta_{0}\right)^{2}} d t, \\
\tan \xi_{n} & =\frac{\Omega\left(v t_{r} / 2\right) \sqrt{n}}{\delta_{0}} \quad \text { with } \xi_{n} \in\left(\frac{-\pi}{2}, \frac{\pi}{2}\right) .
\end{aligned}
$$

We recognize in this expression the central resonant interaction evolution operator, $\mathbf{Y}\left(\theta_{\mathbf{N}}^{r}\right)$, and the two phaseshifts accumulated during the non-resonant interactions, $\left[\mathbf{Z}\left(-\phi_{\mathbf{N}}\right)\right.$ and $\left.\mathbf{Z}\left(\phi_{\mathbf{N}}\right)\right]$. Note that here, unlike in Section $\mathrm{IV}, \phi_{n}$ is a nonlinear function of $n$. The two remaining operators, $\mathbf{X}\left(\xi_{\mathbf{N}}\right)$, reflect the fact that the atomic energy eigenstates do not coincide with the dressed levels at $\pm t_{r} / 2$, when the atomic transition frequency is suddenly switched. Note that we neglect two similar transformations which appear in principle when the atom gets first coupled to the mode and finally decoupled from it, since the atom-field coupling is then quite negligible.

Some tedious but simple computations exploiting Eq. (7) allow us to write:

$$
\begin{aligned}
\mathbf{U}_{c}= & |g\rangle\left\langle g\left|\cos \left(\theta_{\mathbf{N}}^{c} / 2\right)+\right| e\right\rangle\langle e| \cos \left(\theta_{\mathbf{N}+\mathbf{I}}^{c} / 2\right) \\
& -|e\rangle\langle g| \mathbf{a} \frac{\sin \left(\theta_{\mathbf{N}}^{c} / 2\right)}{\sqrt{\mathbf{N}}} e^{i \phi_{\mathbf{N}}^{c}} \\
& +|g\rangle\langle e| \frac{\sin \left(\theta_{\mathbf{N}}^{c} / 2\right)}{\sqrt{\mathbf{N}}} e^{-i \phi_{\mathbf{N}}^{c}} \mathbf{a}^{\dagger} .
\end{aligned}
$$

Here, $\theta_{n}^{c} \in[0,2 \pi)$ is defined by

$$
\cos \left(\theta_{n}^{c} / 2\right)=\cos \left(\theta_{n}^{r} / 2\right) \cos \xi_{n} .
$$

Introducing [39]

$$
\chi_{n}^{c}=\operatorname{angle}\left[\sin \left(\theta_{n}^{r} / 2\right)-i \cos \left(\theta_{n}^{r} / 2\right) \sin \xi_{n}\right]
$$

we define the composite phase as $\phi_{\mathbf{N}}^{c}=\phi_{\mathbf{N}}+\chi_{\mathbf{N}}^{c}$.
Comparing Eqs. (28) and (22), we finally get:

$$
\mathbf{U}_{c}=\mathbf{Z}\left(-\phi_{\mathbf{N}}^{c}\right) \mathbf{Y}\left(\theta_{\mathbf{N}}^{c}\right) \mathbf{Z}\left(\phi_{\mathbf{N}}^{c}\right) \text {. }
$$

This expression of $\mathbf{U}_{c}$ has the same general form as that used in the dispersive case (Section IV). Angles $\theta_{\mathbf{N}}^{c}, \phi_{\mathbf{N}}^{c}$ replace $\theta_{\mathbf{N}}^{r}, \phi_{\mathbf{N}}^{d}$ respectively. We now show that with these adaptations, most of the conclusions of the previous Sections still hold. In particular, each nonclassical state of the form $|\psi\rangle \approx e^{-i t_{K} \mathbf{H}_{K}}|\alpha\rangle$ can indeed be stabilized by a realistic reservoir.

\section{A. Effects of $\mathbf{Y}\left(\theta_{\mathbf{N}}^{c}\right)$ and $\mathbf{Z}\left( \pm \phi_{\mathbf{N}}^{c}\right)$}

Let us first consider a reservoir of atoms whose interaction with the cavity would be described by a propagator $\mathbf{Y}\left(\theta_{\mathbf{N}}^{c}\right)$. Note that this situation is not physical: the $\mathbf{Y}\left(\theta_{\mathbf{N}}^{c}\right)$ evolution operator is no more than a convenient mathematical factor appearing in the expression of the complete evolution operator $\mathbf{U}_{c}$.

In analogy with Section III, the pointer state $\left|\psi_{\infty}\right\rangle=$ $\sum \psi_{n}|n\rangle$ corresponding to this fictitious interaction is defined by the recurrence relation:

$$
\psi_{n+1}=\frac{\tan (u / 2)}{\tan \left(\theta_{n+1}^{c} / 4\right)} \psi_{n},
$$

for $n=0,1,2, \ldots$. Equation (29) ensures $\left|\cos \left(\theta_{n+1}^{c} / 2\right)\right|<$ $1 \forall n$, therefore $0<\theta_{n+1}^{c} / 4<\pi / 2$. Moreover $\lim _{n \mapsto+\infty} \theta_{n}^{c}=\pi$, such that Eq. (32) always defines a unique (normalized) finite-energy state as soon as $|\tan (u / 2)|<1$ i.e. $|u|<\pi / 2$. Indeed, for large $n$ values, Eq. (32) can be approximated by $\psi_{n+1}=\tan \frac{u}{2} \psi_{n}$, showing that $\psi_{n}$ exponentially converges towards 0 and that $\sum_{n} n \psi_{n}^{2}$ is finite. The energy exchange resulting from the fast commutation of the atomic frequency near the cavity center thus removes the possibility of trapping states.

Note that even in the absence of the central resonant interaction, with $\theta_{n}^{r}=0$ in Eq. (29), relation (32) defines a unique pointer state with finite energy. It is thus in principle possible to simplify our scheme by using only two dispersive interactions with opposite detuning.

For $\theta_{r}$ and $\Omega_{r} / \delta_{0}$ small, we get $2 \tan \left(\theta_{n}^{c} / 4\right) \approx \theta_{n}^{c} / 2 \approx$ $\theta_{c} \sqrt{n} / 2$ with $\theta_{c}=\sqrt{\theta_{r}^{2}+\left(\frac{2 \Omega_{r}}{\delta_{0}}\right)^{2}}$. The pointer state is thus close to a coherent state $\left|\alpha_{\infty}\right\rangle$, as in Section III, with the modified amplitude $\alpha_{\infty}=4 \tan (u / 2) / \theta_{c}$. Convergence arguments similar to those of Section III (effective Lindblad master equation) can be given. The convergence rate is now proportional to $\theta_{c}^{2}$. We conjecture that this convergence is valid for any $u$ with $0 \leq u<\pi / 2$, $\theta_{r} \geq 0, \Omega_{r}>0$ and $\delta_{0}>0$.

Figure 5 presents numerical estimations of the field pointer state $\left|\psi_{\infty}\right\rangle$ stabilized by a hypothetical reservoir using interaction $\mathbf{Y}\left(\theta_{\mathbf{N}}^{c}\right)$. All parameter values in the shaded areas lead to at least $99 \%$ fidelity $\left|\left\langle\psi_{\infty} \mid \alpha_{*}\right\rangle\right|^{2}$ w.r.t. a coherent state $\left|\alpha_{*}\right\rangle$ of the same mean photon 

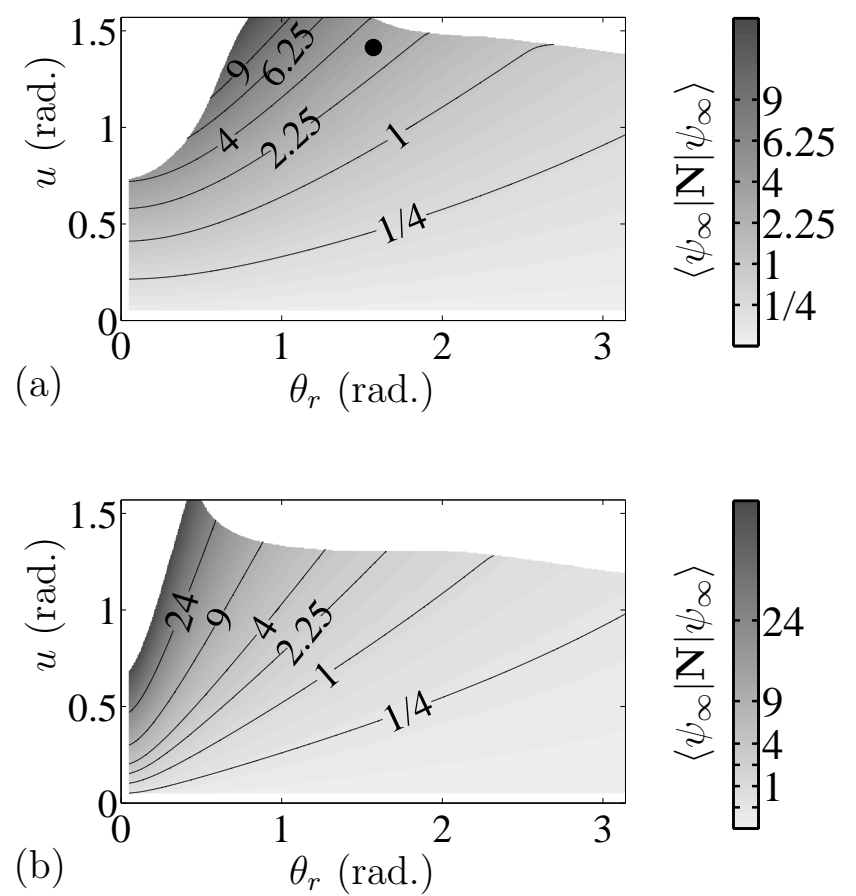

FIG. 5. Mean photon number $\left\langle\psi_{\infty}|\mathbf{N}| \psi_{\infty}\right\rangle$ of the pointer state $\left|\psi_{\infty}\right\rangle$ stabilized by $\mathbf{Y}\left(\theta_{\mathbf{N}}^{c}\right)$, with $\delta_{0}=2.2 \Omega_{r}$ (a) and $\delta_{0}=10 \Omega_{r}$ (b). The grayscale axis is linear in $\sqrt{\left\langle\psi_{\infty}|\mathbf{N}| \psi_{\infty}\right\rangle}$. The 2-component MFSS on Fig. 1 corresponds to $u=0.9 \pi / 2$ and $\theta_{r}=\pi / 2$ with $\delta_{0}=2.2 \Omega_{r}$ [black dot on (a)], for which $\left\langle\psi_{\infty}|\mathbf{N}| \psi_{\infty}\right\rangle=2.96$. The shaded zone is delimited such that all states have at least $99 \%$ fidelity $\left|\left\langle\psi_{\infty} \mid \alpha_{*}\right\rangle\right|^{2}$ to a coherent state $\left|\alpha_{*}\right\rangle$ of the same mean photon number $\left(\left|\alpha_{*}\right|^{2}=\left\langle\psi_{\infty}|\mathbf{N}| \psi_{\infty}\right\rangle\right)$.

number $\left(\left|\alpha_{*}\right|^{2}=\left\langle\psi_{\infty}|\mathbf{N}| \psi_{\infty}\right\rangle\right)$. This mean photon number is represented as a function of parameter values in grayscale and level curves. The smaller parameter value $\Omega_{r} / \delta_{0}=1 / 10$ chosen for Fig. 5 (b) allows to reach higher mean photon numbers than the larger $\Omega_{r} / \delta_{0}=1 / 2.2$ of Fig. 5(a). A reservoir with small $\Omega_{r} / \delta_{0}$ however is also more subject to undesired population of high-number Fock states, reminiscent of the trapping states, for large $\theta_{r}$ and $u$. This explains the smaller domain where fidelity is larger than $99 \%$ on Fig. 5(b). The particular parameter values used for Fig. 1(e) [black dot on Fig. 5(a), with $\Omega_{r} / \delta_{0}=1 / 2.2, \theta=\pi / 2$ and $\left.u=0.45 \pi\right]$ yield a fidelity of almost $99.9 \%$ w.r.t. a coherent state and a mean photon number $\left\langle\psi_{\infty}|\mathbf{N}| \psi_{\infty}\right\rangle=2.96$.

We now examine the influence of the $\mathbf{Z}\left( \pm \phi_{\mathbf{N}}^{c}\right)$ operators on the pointer state defined by $\mathbf{Y}\left(\theta_{\mathbf{N}}^{c}\right)$. A first observation is that it does not modify the photon number populations, since it commutes with $\mathbf{N}$. Thus, the energy of the field pointer state, for a reservoir with composite interaction, is entirely determined by $\mathbf{Y}\left(\theta_{\mathbf{N}}^{c}\right)$, as represented for example on Fig. 5. Let us define the Hermitian operator $h_{\mathbf{N}}^{c}$ by the recurrence relation:

$$
h_{n+1}^{c}-h_{n}^{c}=\phi_{n+1}^{c},
$$

for $n=0,1,2, \ldots$, with an arbitrary $h_{0}^{c}$. Using Eq. (7) as in Section IV yields

$$
\mathbf{U}_{c}=e^{-i h_{\mathbf{N}}^{c}} \mathbf{Y}\left(\theta_{\mathbf{N}}^{c}\right) e^{i h_{\mathbf{N}}^{c}}
$$

The pointer states of $\mathbf{U}_{c}$ are thus obtained by applying the unitary transformation $e^{-i h_{\mathbf{N}}^{c}}$ to the pointer states of $\mathbf{Y}\left(\theta_{\mathbf{N}}^{c}\right)$. The choice of $h_{0}^{c}$ for solving Eq. (33) is physically irrelevant, as it corresponds to a global phase factor that cancels out in Eq. (34). The operator $h_{\mathbf{N}}^{c}$ here exactly plays the role of $h_{\mathbf{N}}^{d}$ in Section IV. The only difference is that, as $\phi_{n}^{c}$ is nonlinear, $h_{n}^{c}$ is defined through the discrete integral (33). If $\phi_{n}^{c}$ is nearly linear in $n$ over the relevant photon numbers [dominant photon numbers in the pointer state $\left|\psi_{\infty}\right\rangle$ associated to $\left.\mathbf{Y}\left(\theta_{\mathbf{N}}^{c}\right)\right]$, then $h_{n}^{c}$ is nearly quadratic. The situation of Section $\mathrm{V}$ is recovered; The reservoir stabilizes nonclassical pointer states $\left|\psi_{\infty}^{c}\right\rangle=e^{-i h_{\mathbf{N}}^{c}}\left|\psi_{\infty}\right\rangle \approx e^{-i t_{K} \mathbf{H}_{K}}|\alpha\rangle$ with $t_{K}$ depending on the parameters governing $\phi_{n}^{c}$.

\section{B. Choice of the reservoir operating point}

We now use the detailed description of the reservoir to justify the choice of operating parameters for generating the two-component MFSS presented in figure 1(e): $u=0.45 \pi, \theta_{r}=\pi / 2, v=70 \mathrm{~m} / \mathrm{s}$ and $\delta=2.2 \Omega_{0}$. Note that the state in Fig. $1(\mathrm{e})$, with $\approx 2.7$ photons on the average, has been computed with a finite cavity lifetime $T_{c}=65 \mathrm{~ms}$ and a random (zero or one) atom number in each atomic sample. The same computation leads to an average photon number equal to 2.96 in an ideal cavity, see Fig. 5. The two-component MFSS requires the largest effect from the dispersive interactions, and hence corresponds to the most demanding experimental conditions.

The chosen parameters are the result of a tradeoff between contradictory requirements. First, the composite phase shift $\phi_{n}^{c}$ must be nearly linear in $n$ over the useful photon number range, with a slope of $\pi$ per photon. Second, the time of convergence towards the steady state needs to be much shorter than its expected decoherence time $\left(T_{c} / 5.6\right)$ associated to cavity relaxation. Linearity of $\phi_{n}^{c}$ improves with larger $\delta_{0} / \Omega_{r}$. But achieving a phase shift of $\pi$ per photon then requires a longer atom-cavity interaction time, which is detrimental for the second requirement.

The tradeoff is further examined on Fig. 6. Figure 6(a) evaluates the linearity of $\phi_{n}^{c}$ by showing $D \phi_{n}^{c}=$ $\phi_{n+1}^{c}-\phi_{n}^{c}=h_{n+1}^{c}+h_{n-1}^{c}-2 h_{n}^{c}$ for different parameter values. The value of $\theta_{r}$ has little influence and we set it to $\pi / 2$. For each value of $\Omega_{r} / \delta_{0}$, we adjust $v$ to have $D \phi_{n}^{c}=\pi$ for the same mean photon number $n=2.96$ (by linear interpolation). This value is chosen to match the parameter values of Fig. 1(e), represented by a black dot on Figs. 5 and 6 . As expected, $D \phi_{n}^{c}$ is quite constant for moderate photon numbers in the dispersive region $\delta_{0} / \Omega_{r} \gg 1$. This corresponds however to prohibitively small atomic velocities, represented on Fig. 6(b). In the region of low $\delta_{0} / \Omega_{r}$ values, a $D \phi_{n}^{c} \approx \pi$ at $n=2.96$ can 
(a)
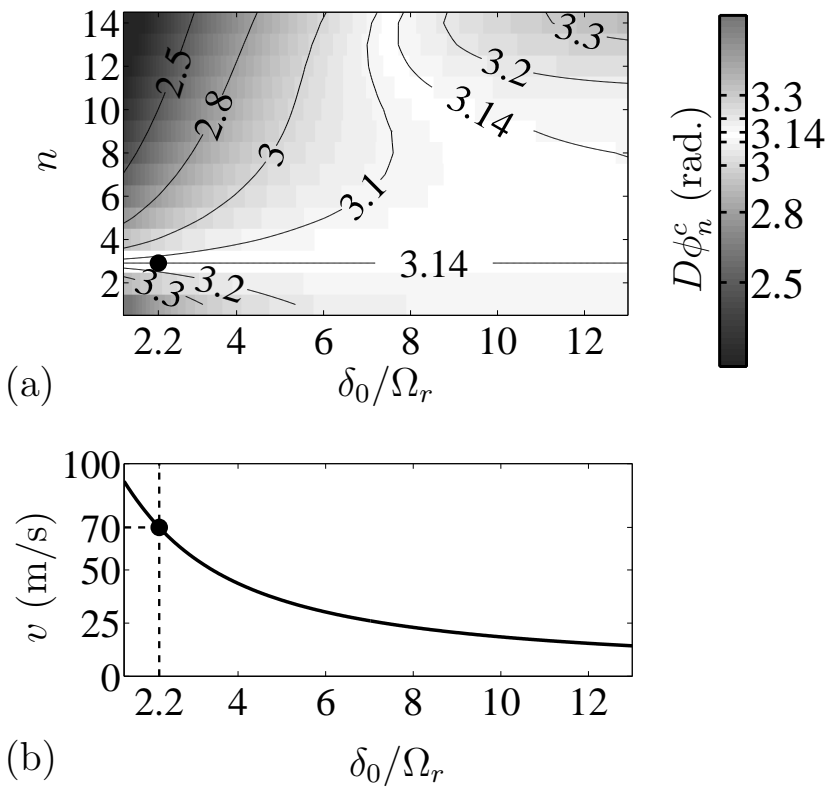

FIG. 6. (a) Kerr-effect-inducing $D \phi_{n}^{c}=\phi_{n+1}^{c}-\phi_{n}^{c}$ as a function of photon number $n$ and $\delta_{0} / \Omega_{r}$. Since $\phi_{0}^{c}$ is undefined, we start with $D \phi_{1}^{c}$. (b) Corresponding velocities $v$ : for each $\delta_{0} / \Omega_{r}$, we adjust $v$ to have $D \phi_{n}^{c}=\pi$ at $n=2.96$. This value is chosen to cover the parameter values $v=70 \mathrm{~m} / \mathrm{s}$, $\delta_{0} / \Omega_{r}=1 / 2.2$ (black dots) used for the 2-component MFSS in Fig. 1. An ideal $h_{n}^{c}$, proportional to $\mathbf{H}_{K}$, requires $D \phi_{n}^{c}$ constant in $n$. Small $\delta_{0} / \Omega_{r}$ values are disadvantageous for this criterion, but makes it possible to use higher velocities and hence more frequent reservoir atoms for a same mean $D \phi^{c}$. We have selected a constant value $\theta_{r}=\pi / 2$ for this figure.

be reached with larger $v$, but $D \phi_{n}^{c}$ varies more rapidly with $n$. This variation is nevertheless sufficiently weak in the range $2 \leq n \leq 5$ for $\delta_{0} / \Omega_{r} \approx 2.2$, corresponding to the velocity $v=70 \mathrm{~m} / \mathrm{s}$ that is used for Fig. 1(e).

Let us now examine the overall reservoir fidelity and the convergence rate $\lambda_{\text {conv }}$ from the vacuum towards the target state, as already defined in Fig. 4. We analyze their dependency on the parameters $\theta_{r}$ and $\delta_{0} / \Omega_{r}$. For each value of $\theta_{r}$ and $\delta_{0} / \Omega_{r}$, we set $v$ to the unique value which corresponds to a $\left|\psi_{\infty}^{c}\right\rangle$ of the form $\left|c_{\alpha}\right\rangle$ [see Fig. 6(b)]. Then $u$ is adjusted so that the target mean photon number is 2.96 (see Fig. 5(a)). Figure 7(a) shows the ratio $\lambda_{\text {conv }} / T$, where $T$ is the total interaction time of each atom with the cavity. This ratio characterizes the convergence rate in $\mathrm{s}^{-1}$ units. The fidelity with respect to an ideal MFSS with the same mean photon number 2.96 is shown on Fig.7(b). The black dot represents the parameter values $\delta_{0} / \Omega_{r}=2.2$ and $\theta_{r}=\pi / 2$ chosen for Fig. 1(e). They do not correspond to a maximum fidelity, because the relatively small value of $\delta_{0} / \Omega_{r}$ induces significant variation of $D \phi_{n}^{c}$ in the useful $n$ value range (see Fig. 6(a)). However, for a still excellent $95 \%$ fidelity, the associated convergence rate $\left(1400 \mathrm{~s}^{-1}\right)$ is high and the reservoir competes efficiently with decoherence
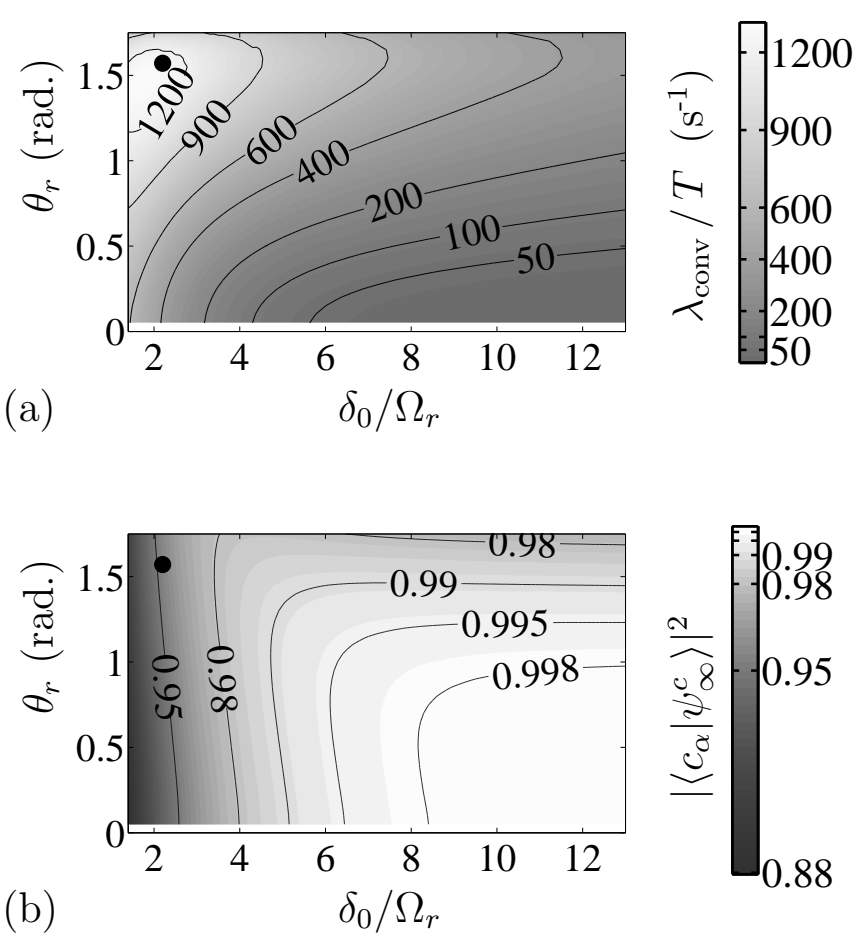

FIG. 7. (a): Convergence rate $\lambda_{\text {conv }} / T$ giving the slope, in time units $\left(\mathrm{s}^{-1}\right)$, of the convergence towards the reservoir pointer state $\left|\psi_{\infty}^{c}\right\rangle$, according to $\log \left|\log \left\langle\psi_{\infty}^{c}\left|\rho_{k}\right| \psi_{\infty}^{c}\right\rangle\right|=$ $\log \left|\log \left\langle\psi_{\infty}^{c}\left|\rho_{0}\right| \psi_{\infty}^{c}\right\rangle\right|-\lambda_{\text {conv }} k$ (see Fig. 4). For each $\theta_{r}$ and $\Omega_{r} / \delta_{0}$, we adjust $v$ as in Fig. 6 to keep $D \phi_{n}^{c} \approx \pi$, and $u$ to keep $\left\langle\psi_{\infty}^{c}|\mathbf{N}| \psi_{\infty}^{c}\right\rangle=2.96$. The choice of these reference values corresponds to $\theta_{r}=\pi / 2, u=0.45 \pi, \Omega_{r} / \delta_{0}=2.2$, $v=70 \mathrm{~m} / \mathrm{s}$ (black dot) used for Fig. 1(e). Time $T=3 w / v$ between consecutive atoms changes as we adjust $v$. (b): Fidelity of the same $\left|\psi_{\infty}^{c}\right\rangle$ to a 2-component MFSS $\left|c_{\alpha_{\infty}}^{\prime}\right\rangle=$ $\left(\left|\alpha_{\infty}\right\rangle+i e^{i \beta}\left|-\alpha_{\infty}\right\rangle\right) / \sqrt{2}$, where we tune $\alpha_{\infty}$ and $0 \leq \beta<\pi$ to optimize fidelity. It turns out that $|\beta|<0.005$ for most parameter values, while $\left|\alpha_{\infty}\right|^{2}$ decreases as fidelity decreases, below 2.7 for the lowest values of $\Omega_{r} / \delta_{0}$. The black dot marks the settings for the 2-component MFSS in Fig. 1. For $\theta_{r}$ values larger than those represented, there is no $u$ value stabilizing a mean photon number 2.96, see also Fig. 5. The two plots together illustrate a tradeoff between fidelity in absence of decoherence and convergence speed.

whose expected time scale is of order of $65 / 5.6 \approx 10 \mathrm{~ms}$ (see next Section). Figure 8 shows the Wigner functions (left) of the steady state MFSS obtained with this parameter choice and (right) of a theoretical superposition of two coherent states with opposite phases and the same total energy. The slight distortions of the quasi-coherent components in the pointer state MFSS are due to the nonlinearity of the phase shift $\phi_{n}^{c}$. 


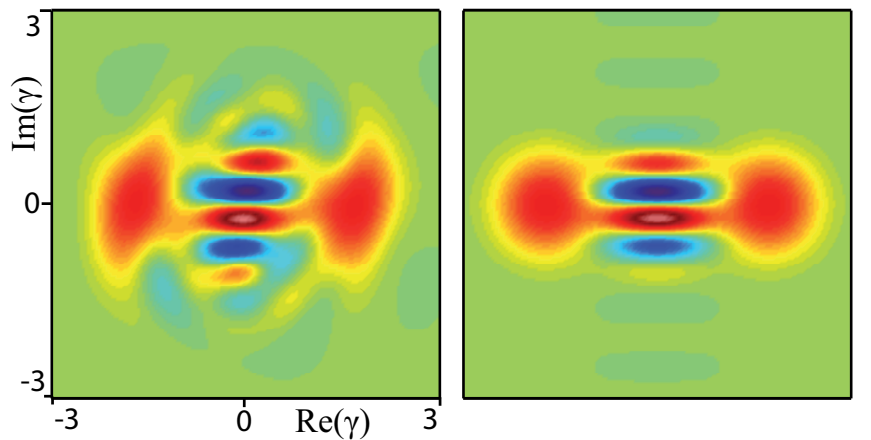

FIG. 8. (Color) Wigner functions $W(\gamma)$ illustrating a stabilized 2-component MFSS (colorbar as in Fig. 1). Left: $\rho=\left|\psi_{\infty}^{c}\right\rangle\left\langle\psi_{\infty}^{c}\right|$, pointer state of our reservoir with composite interaction. Parameter values are $\theta_{r}=\pi / 2, u=0.45 \pi$, $\delta_{0}=2.2 \Omega_{0}, v=70 \mathrm{~m} / \mathrm{s}$, i.e. those used for figure $1(\mathrm{e})$, except $T_{c}$ set to infinity here. Right: target state $\rho=\left|c_{\alpha}\right\rangle\left\langle c_{\alpha}\right|$.

\section{DECOHERENCE AND EXPERIMENTAL IMPERFECTIONS}

The above choice of operating parameters has been based on a rough estimate of the action of decoherence. We now explicitly show how the reservoir allows us to stabilize a Mesoscopic Field State Superposition with a high fidelity, in the presence of cavity relaxation into a zero-temperature environment (Section VI A). In Section VIB we study the robustness of the scheme against other experimental imperfections by numerical simulations.

\section{A. Cavity relaxation}

We first analyze the field evolution with a simplified model. The simplified model without relaxation is obtained from equation (19) for the coherent state evolution, sandwiched between two dispersive transformations (Eq. (21)):

$$
\begin{aligned}
\alpha_{k+1} & =\left(1-\theta_{r}^{2} / 8\right) \alpha_{k}+u \theta_{r} / 4 \\
\rho_{k}^{\prime h} & =\left|\alpha_{k}\right\rangle\left\langle\alpha_{k}\right| \\
\rho_{k}^{\prime} & =e^{-i \pi / 2 \mathbf{N}^{2}} \rho_{k}^{\prime h} e^{i \pi / 2 \mathbf{N}^{2}} .
\end{aligned}
$$

In a Monte-Carlo approach, the evolution of the field density matrix due to cavity relaxation can be represented as a succession of quantum jumps described by the annihilation operator a, occurring at random times and interrupting a non-unitary deterministic evolution close to (35) [40].

The action of $\mathbf{a}$ on an MFSS $\left|c_{\alpha}\right\rangle$ writes:

$$
\mathbf{a}\left|c_{\alpha}\right\rangle \propto\left|c_{-\alpha}\right\rangle
$$

This loss of photons in the environment cannot be detected. Therefore, in the absence of the reservoir, an initial state $\left|c_{\alpha}\right\rangle$ would rapidly evolve into a statistical mixture of $\left|c_{\alpha}\right\rangle$ and $\left|c_{-\alpha}\right\rangle$, i.e. into a mixture of $|\alpha\rangle$ and $|-\alpha\rangle$. The purpose of the reservoir is to drive $\left|c_{-\alpha}\right\rangle$ back to $\left|c_{\alpha}\right\rangle$ after each jump. If the reservoir-induced convergence time is much shorter than the average interval between two jumps, then the field is mostly close to $\left|c_{\alpha}\right\rangle$.

This simple description suggests to seek a steady state of the field evolution, driven by the engineered reservoir and the cavity relaxation, in the form $\rho_{\infty}^{\prime h}=$ $\int \mu(z)|z\rangle\langle z| d z$. This is a statistical mixture of coherent states $|z\rangle$ with a real amplitude $z$ weighted by $\mu(z)$.

In the absence of cavity relaxation, the evolution of $\rho_{k}^{\prime h}$ in the simplified model [Eq. (35)] can also be viewed as a discretization of the Lindblad master equation:

$$
\frac{d}{d t} \rho^{\prime h}=\left[\beta \mathbf{a}^{\dagger}-\beta^{\dagger} \mathbf{a}, \rho^{\prime h}\right]-\frac{\kappa}{2}\left(\mathbf{N} \rho^{\prime h}+\rho^{\prime h} \mathbf{N}-2 \mathbf{a} \rho^{\prime h} \mathbf{a}^{\dagger}\right),
$$

with $\beta d t=u \theta_{r} / 4$ and $\kappa d t=\theta_{r}^{2} / 4$. An equation of the same form as (36) also describes the evolution of a field mode coupled with a classical source of amplitude $\beta$ and damped at a rate $\kappa$. In these conditions, $\rho^{\prime h}$ converges towards a coherent state $\left|\alpha_{\infty}\right\rangle$ with $\alpha_{\infty}=2 \beta / \kappa$, see e.g. [4]. Note that in the situation described by Eq.(36), the damping rate $\kappa$ is induced by the atomic reservoir and not by cavity relaxation. As $\rho^{\prime h}$ follows (36), $\rho^{\prime}=e^{-i \pi / 2 \mathbf{N}^{2}} \rho^{\prime h} e^{i \pi / 2 \mathbf{N}^{2}}$ evolves according to:

$$
\begin{aligned}
\frac{d}{d t} \rho^{\prime}=\beta\left[\mathbf{a}^{\dagger} e^{-i \pi \mathbf{N}}-e^{i \pi \mathbf{N}} \mathbf{a}, \rho^{\prime}\right] & \\
& -\frac{\kappa}{2}\left(\mathbf{N} \rho^{\prime}+\rho^{\prime} \mathbf{N}-2 e^{i \pi \mathbf{N}} \mathbf{a} \rho^{\prime} \mathbf{a}^{\dagger} e^{-i \pi \mathbf{N}}\right),
\end{aligned}
$$

where we can assume, up to a phase reference choice, that $\beta$ is real and positive.

It is now straightforward to add, to this simplified continuous-time model, the usual Lindblad terms which describe the action of a thermal environment at zero temperature, inducing decoherence of the field with a finite cavity lifetime $T_{c}=1 / \kappa_{c}$ :

$$
\begin{aligned}
\frac{d}{d t} \rho^{\prime}=\beta\left[\mathbf{a}^{\dagger} e^{-i \pi \mathbf{N}}-\right. & \left.e^{i \pi \mathbf{N}} \mathbf{a}, \rho^{\prime}\right] \\
-\frac{\kappa}{2}\left(\mathbf{N} \rho^{\prime}+\right. & \left.\rho^{\prime} \mathbf{N}-2 e^{i \pi \mathbf{N}} \mathbf{a} \rho^{\prime} \mathbf{a}^{\dagger} e^{-i \pi \mathbf{N}}\right) \\
& -\frac{\kappa_{c}}{2}\left(\mathbf{N} \rho^{\prime}+\rho^{\prime} \mathbf{N}-2 \mathbf{a} \rho^{\prime} \mathbf{a}^{\dagger}\right) .
\end{aligned}
$$

The associated $\rho^{\prime h}$ in the Kerr representation then evolves according to:

$$
\begin{aligned}
\frac{d}{d t} \rho^{\prime h}=\beta\left[\mathbf{a}^{\dagger}-\mathbf{a}, \rho^{\prime h}\right] & \\
-\frac{\kappa+\kappa_{c}}{2} & \left(\mathbf{N} \rho^{\prime h}+\rho^{\prime h} \mathbf{N}-2 \mathbf{a} \rho^{\prime h} \mathbf{a}^{\dagger}\right) \\
& -\kappa_{c}\left(\mathbf{a} \rho^{\prime h} \mathbf{a}^{\dagger}-e^{i \pi \mathbf{N}} \mathbf{a} \rho^{\prime h} \mathbf{a}^{\dagger} e^{-i \pi \mathbf{N}}\right) .
\end{aligned}
$$

Without the terms in the third line, Eq. (38) would be identical to Eq. (36) with $\kappa$ replaced by $\kappa+\kappa_{c}$. This would simply drive $\rho^{\prime h}$ to a coherent steady state of reduced amplitude $\alpha_{\infty}^{c}=\alpha_{\infty} /(1+\eta)$ with $\eta=(4 T) /\left(\theta_{r}^{2} T_{c}\right)$. We then observe that the whole equation (38) leaves invariant the set of all mixtures of coherent states with real amplitudes in $\left[-\alpha_{\infty}^{c}, \alpha_{\infty}^{c}\right]$. Therefore, we search for a steady state of Eq. (38) under the form:

$$
\rho_{\infty}^{\prime h}=\int_{-\alpha_{\infty}^{c}}^{\alpha_{\infty}^{c}} \mu(z)|z\rangle\langle z| d z .
$$


As explained in the Appendix B, this yields a solution:

$$
\mu(z)=\mu_{0} \frac{\left(\left(\left(\alpha_{\infty}^{c}\right)^{2}-z^{2}\right)^{\left(\alpha_{\infty}^{c}\right)^{2}} e^{z^{2}}\right)^{r_{c}}}{\alpha_{\infty}^{c}-z}
$$

with $r_{c}=2 \kappa_{c} /\left(\kappa+\kappa_{c}\right)$. The normalization factor $\mu_{0}>0$ ensures that $\int_{-\alpha_{\infty}^{c}}^{\alpha_{\infty}^{c}} \mu(z) d z=1$. In any case, $\mu\left(-\alpha_{\infty}^{c}\right)=$ 0 . For small $\kappa_{c}$, we have $\lim _{z \mapsto \alpha_{\infty}^{c}} \mu(z)=+\infty$ and $\rho_{\infty}^{\prime h}$ is close to the coherent state $\left|\alpha_{\infty}^{c}\right\rangle$. For large $\kappa_{c}, \alpha_{\infty}^{c}$ is anyway close to zero and the field steady-state comes close to the vacuum.

We now compare this simplified model to the actual reservoir in the presence of relaxation. Figure 9 illustrates the reservoir-induced convergence after a quantum jump. The leftmost column shows the Wigner function of $\rho^{\prime}$, evolving under the simplified model (35). Starting at $\left|c_{\alpha}\right\rangle$ (upper left frame), we assume that a decoherenceinduced jump immediately takes the state to $\left|c_{-\alpha}\right\rangle$ (second frame in the leftmost column). The successive snapshots on the next frames illustrate how the state gradually converges back towards $\left|c_{\alpha}\right\rangle$ under the dynamics described by Eq. (35). We neglect here the action of cavity relaxation during this recovery process. Note that after $\approx 4$ reservoir atoms, a vacuum state is reached, from which $\left|c_{\alpha}\right\rangle$ is gradually recovered.

The second column depicts the evolution of $\rho^{\prime h}$. In this representation, the initial state is the coherent state $|\alpha\rangle$ (first frame). It jumps to $|-\alpha\rangle$ (second frame), and then gradually evolves back towards $|\alpha\rangle$ according to Eq. (19), staying coherent at all times.

On the third column, we show the Wigner functions of the actual cavity state $\rho$ induced by our reservoir, whose dynamics is governed by the Kraus map associated to $\mathbf{U}_{c}$. The last column shows the evolution of $\rho^{h}=e^{i h_{\mathbf{N}}^{c}} \rho e^{-i h_{\mathbf{N}}^{c}}$. We observe that $\rho^{h}$ and $\rho^{\prime h}$ follow qualitatively the same path. Our analysis on the simplified model thus seems to describe the actual cavity state evolution in the presence of relaxation reasonably well. The main difference is a notable distortion of $\rho^{h}$ when the field amplitude is near zero.

We can further examine the steady states $\rho_{\infty}^{\prime}$ and $\rho_{\infty}$ of the (simplified and actual) reservoirs in the presence of relaxation, that approximately correspond to the quantum Monte-Carlo average of the trajectories depicted in Fig. 9. Figure 10 shows the marginal distributions, along the real and imaginary quadratures, of the Wigner functions for $\rho_{\infty}^{\prime h}$ and $\rho_{\infty}^{h}$. They feature dominant peaks which suggest that the field is mostly close to the target. The distortions with respect to a coherent state visible on the fourth column of Fig. 9, lead to a plateau or bump on the marginal distributions of $\rho_{\infty}^{h}$. We nevertheless observe that our simplified model [Eq.(37)] captures the main features of the influence of decoherence.

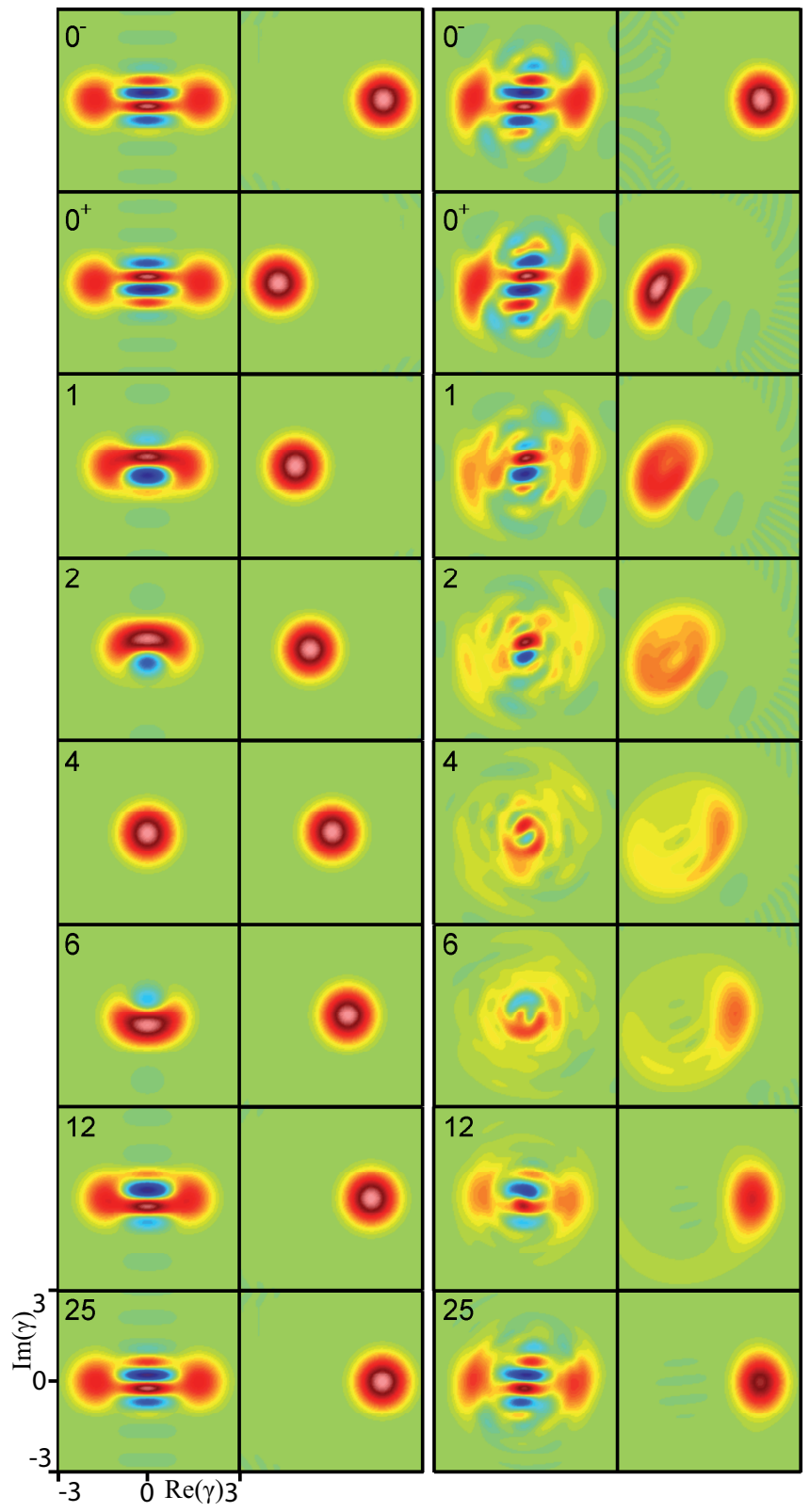

FIG. 9. (Color) Evolution of the cavity field coupled to a reservoir stabilizing a 2-component MFSS, immediately after a relaxation-induced photon loss. We use the same parameter values as for Fig. 8. A photon loss out of the reservoir pointer state occurs between the frames labelled $0^{-}$and $0^{+}$. The other frames are labelled by the number of atomic interactions, that have taken place after the photon loss, with no further relaxation. Left two columns: simplified model, described by Eq. (35). We show the Wigner functions of the cavity state, $\rho^{\prime}$, on column 1 and of $\rho^{\prime h}$ on column 2 . Right two columns: same plots for the actual reservoir characterized by $\mathbf{U}_{c}$ ( $\rho$ on column 3 and $\rho^{h}$ on column 4 ).

\section{B. Experimental uncertainties}

We have performed extensive numerical simulations to assess the robustness of the reservoir towards uncertain- 

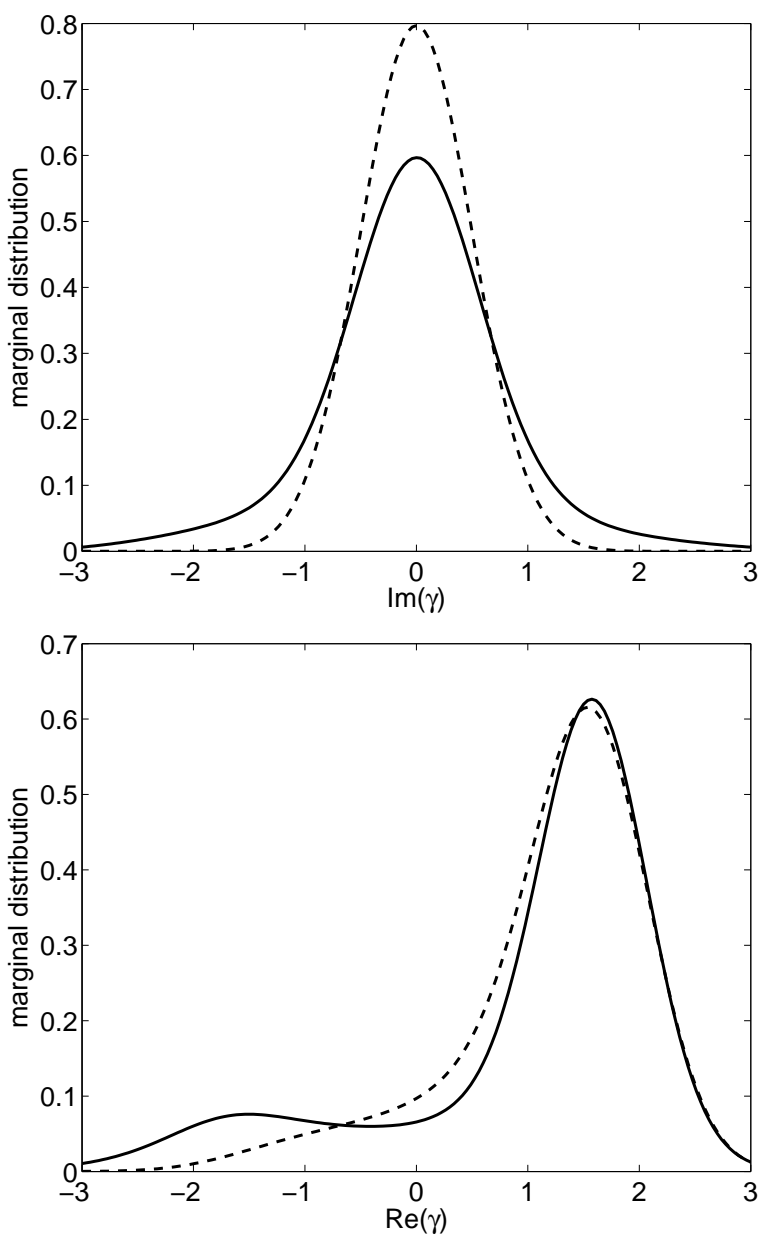

FIG. 10. Steady state of the cavity field coupled to the atomic reservoir and to a relaxation-inducing environment with $T_{c}=65 \mathrm{~ms}$. Top [resp: bottom]: marginal distribution of the Wigner function along the imaginary [resp: real] quadrature for the simplified model $\rho_{\infty}^{\prime h}$ (dashed line) and for our reservoir $\rho_{\infty}^{h}$ (solid line). These states approximately correspond to the quantum Monte-Carlo average of the sequence presented in Fig. 9.

ties in the experimental setup. For these simulations, the evolution operators associated to the reservoir are computed by exactly integrating the Hamiltonian $\mathbf{H}_{J C}$ [Eq. (3)], using the quantum optics package for MATLAB [41]. The Hilbert space is truncated to the 60 first Fock states. We separately take into account the atomcavity coupling ruled by $\mathbf{H}_{J C}$ and the relaxation of the cavity mode, modeled in the standard Lindblad form. This simplification holds since $T \ll T_{c}$.

We use as a reference the generation of a twocomponent MFSS containing 2.7 photons on the average [thermal environment with $T_{c}=65 \mathrm{~ms}$ and a mean number $n_{t}=0.05$ of blackbody photons per mode, $\delta=2.2 \Omega_{0}$, $v=70 \mathrm{~m} / \mathrm{s}, u=0,45 \pi, t_{r}=5 \mu \mathrm{s}$, see Fig. 1(e)]. We also take into account the randomness of the Rydberg state preparation [32]. In fact, assuming that precisely one atom interacts with the field at each iteration is an un- realistic simplification for the ENS experiment. Instead, we can only prepare atomic samples with a random number of atoms, obeying a Poisson law of average $p_{a t}$. We select a low value $p_{a t}=0.3$ such that, in a first approximation, we only get samples containing either no atom or exactly one (we examine later in this Section the influence of samples containing two atoms). Note that these are the conditions used for figure 1(e). For this reference set of parameters, the steady-state fidelity w.r.t. an ideal optimized two-component MFSS is 70\%. We now examine how this fidelity is affected by various small variations in the experimental setting.

We have first checked that the atomic velocity dispersion expected in the experiment - that is well below $10 \%$ - has nearly no effect on the fidelity. Indeed, for $v$ varying between 66 and $74 \mathrm{~m} / \mathrm{s}$, the fidelity remains within $65 \%$ to $70 \%$.

The fidelity is also quite insensitive to a slight mismatch in the values of the detuning for the two dispersive interactions. Assuming that $\delta$ takes the value $a_{1} \times 2.2 \Omega_{0}$ in the first dispersive period and $-a_{2} \times 2.2 \Omega_{0}$ in the second, the fidelity drops by at most $10 \%$ when $a_{1}$ and $a_{2}$ vary by up to $\pm 10 \%$. This range of parameters covers far more than the actual uncertainty on the atomic frequency.

Imprecisions in the timing of the detuning profile are expected to have similar effects. In fact, we observe that shifting the resonant interaction in time by up to $1 \mu \mathrm{s}$ (well above the experimental $10 \mathrm{~ns}$ timing accuracy) affects the fidelity by less than $1 \%$. The finite rise time for the voltage controlling the atomic Stark effect in the cavity also induces a transient evolution of $\delta$ between the consecutive detuning values. Using an exponential model for convergence to the new $\delta$ value, and adjusting $t_{r}$ to maintain a constant $\theta_{r}$ value, we find that the fidelity is unchanged for commutation times up to a realistic $200 \mathrm{~ns}$ value.

We have also studied the effect of atomic samples containing two atoms at the same time. For a two-atom sample, we integrate the exact equations of motion, assuming an identical coupling of both atoms to the mode. This condition is realized in the experiment, since the maximum separation between the atoms in a sample is, below $1 \mathrm{~mm}$ in $C$, a value much smaller than the wavelength $-6 \mathrm{~mm}-$ or than the mode waist $w$. We observe that the two-atom events do have an impact on the fidelity. If we consider an unrealistic reservoir involving samples containing always two atoms, we would get as a steady-state a large two-component cat, with 4.8 photons on the average and a fidelity of $65 \%$. In the real situation, this two-atom engineered reservoir interferes destructively with the operation of the one-atom samples.

We have therefore computed the expected steady state fidelity when the actual number of atoms $N_{a}$ in each sample is randomly chosen according to a Poisson distribution with the average value $p_{a t}$ and truncated above $N_{a}=2$. For $p_{a t}=0.3$, the energy of the prepared cat 
decreases down to 2.4 photons on the average and the fidelity is reduced to $66 \%$. For larger $p_{a t}$ values, the decrease is more important; The fidelity reduces to $34 \%$ for $p_{a t}=0.5$ (for even larger $p_{a t}$, the simulation should also include 3 -atom samples). When we reduce $p_{a t}$ below 0.3 , the fidelity and the energy also decrease, since the reservoir is then less efficient to counteract decoherence. For $p_{a t}=0.2$ for instance, we get a 1.9 photons state with a fidelity of only $54 \%$. Optimizing the average number of atoms per sample is thus important to achieve an efficient engineered reservoir.

Note finally that the phase of the MFSS coherent components is determined by the phase of the atomic state superposition when the resonant interaction begins. Since the atom is detuned from $\omega_{c}$ during the dispersive interactions, this phase rotates at the frequency $\delta_{0}$ during the time interval $-T / 2 \leq t \leq-t_{r} / 2$. The timing of the Stark shifts, that govern the atom-field interactions, should thus define $\left(T-t_{r}\right) / 2$ with an uncertainty much smaller than $1 / \delta_{0}$ to avoid spurious rotations of this phase. With detuning values in the $100 \mathrm{kHz}$ to few $\mathrm{MHz}$ range, the required timing accuracy is easily achieved.

\section{A RESERVOIR FOR TWO-MODE ESMS}

Our reservoir engineering strategy can be adapted to protect entangled state superpositions of two cavity modes, which violate a Bell inequality. The preparation of entangled states of two cavity modes, without protection, has been considered in [6, 42]. An approximate reservoir for entangling large atomic ensembles is proposed and realized in [27]. In ion traps, reservoir engineering has recently been used to stabilize a Bell state and a GHZ state of four qubits [26].

We here present a scheme in which the two modes belong to the same cavity (two TEM modes of orthogonal polarization, whose degeneracy is lifted by an appropriate mirror shape). Extension to two separate cavities would require atoms going back and forth between the cavities, a feat not easily achieved in the ENS experiment. We anticipate that other experimental settings might overcome this obstacle.

\section{A. Model and target}

We consider two modes $a$ and $b$ of the cavity, with respective frequencies $\omega_{a}<\omega_{b}$, interacting with atomic qubits of transition frequency $\omega_{0} \approx \omega_{a}, \omega_{b}$. We note $\mathbf{b}$ [resp. a] the photon annihilation operator for mode $b$ $[$ resp. mode $a]$ and $\mathbf{N}_{b}=\mathbf{b}^{\dagger} \mathbf{b}\left[\right.$ resp. $\left.\mathbf{N}_{a}=\mathbf{a}^{\dagger} \mathbf{a}\right]$ the associated photon number operator. A separable joint state of the two modes is written $\left|\psi_{a}, \psi_{b}\right\rangle$. We describe the states in a frame rotating at the frequency $\omega_{m}=\left(\omega_{a}+\omega_{b}\right) / 2$, such that the free field evolution follows the Hamiltonian $\overline{\mathbf{H}}_{f}=\Delta\left(\mathbf{N}_{b}-\mathbf{N}_{a}\right)$ with $\Delta=\left(\omega_{b}-\omega_{a}\right) / 2>0$.
The Jaynes-Cummings Hamiltonian describing the interaction of one atomic qubit with the modes then writes (with the standard approximations):

$$
\begin{aligned}
\overline{\mathbf{H}}_{J C}= & \Delta\left(\mathbf{N}_{b}-\mathbf{N}_{a}\right)+\frac{\delta(t)}{2}(|e\rangle\langle e|-| g\rangle\langle g|) \\
& +i \frac{\Omega(s)}{2}\left(|g\rangle\left\langle e\left|\left(\mathbf{a}^{\dagger}+\mathbf{b}^{\dagger}\right)-\right| e\right\rangle\langle g|(\mathbf{a}+\mathbf{b})\right),
\end{aligned}
$$

where $\delta(t)=\omega_{0}(t)-\omega_{m}$ can again be adjusted by controlling $\omega_{0}$ through the Stark effect. Here, we assume that the coupling $\Omega(s)$ is the same with both modes, a restriction that could easily be relaxed.

We note $\overline{\mathbf{U}}$ the unitary evolution operator associated to $\overline{\mathbf{H}}_{J C}$ (the overline characterizes two-mode operators), solution of the Schrödinger equation:

$$
\frac{d}{d t} \overline{\mathbf{U}}(t)=-i \overline{\mathbf{H}}_{J C}(t) \overline{\mathbf{U}}(t) \quad \text { with } \overline{\mathbf{U}}\left(t_{0}\right)=\mathbf{I} .
$$

We note $\overline{\mathbf{U}}_{q}$ the two-mode evolution operator corresponding to the parameter set $q$, and $\left(\mathbf{M}_{g}^{\overline{\mathbf{U}}_{q}}, \mathbf{M}_{e}^{\overline{\mathbf{U}}_{q}}\right)$ the associated Kraus operators. Approximate analytical expressions of $\overline{\mathbf{U}}_{q}$ for relevant parameter sets are given in Appendix A. This appendix also formally defines operators $\overline{\mathbf{Z}}$ and $\overline{\mathbf{Y}}$ that generalize the single-mode operators introduced in the previous Sections.

Let us first consider a situation where each atom, initially prepared in $\left|u_{a t}\right\rangle=\cos (u / 2)|g\rangle+\sin (u / 2)|e\rangle$, undergoes a resonant interaction with mode $b$ followed by a resonant interaction with mode $a$. The corresponding propagator is $\overline{\mathbf{U}}_{r}=\overline{\mathbf{Y}}\left(\theta_{\mathbf{N}_{a}}^{r}\right) \overline{\mathbf{Y}}\left(\theta_{\mathbf{N}_{b}}^{r}\right)$. The associated Kraus map $\left(\mathbf{M}_{g}^{\overline{\mathbf{U}}_{r}}, \mathbf{M}_{e}^{\overline{\mathbf{U}}_{r}}\right)$ stabilizes a tensor product of two coherent states $|-\alpha, \alpha\rangle$, where $\alpha=2 u / \theta_{r}$ for small enough $u$ and $\theta_{r}$.

The action of the Kerr-like Hamiltonian

$$
\overline{\mathbf{H}}_{K}=-\bar{\gamma}_{K}\left(\left(\mathbf{N}_{a}+\mathbf{N}_{b}\right)^{2}+2 \mathbf{N}_{a}\right)
$$

for a time $t_{K}=\frac{\pi}{2 \bar{\gamma}_{K}}$ would transform an initial state $|-\alpha, \alpha\rangle$ into:

$$
\left|\bar{c}_{\alpha}\right\rangle=(|\alpha, \alpha\rangle-i|-\alpha,-\alpha\rangle) / \sqrt{2} .
$$

In the next Section, we show that the action of $\overline{\mathbf{H}}_{K}$ can be simulated by sandwiching the resonant interaction $\overline{\mathbf{U}}_{r}$ between two dispersive interactions. The corresponding reservoir thus stabilizes $\left|\bar{c}_{\alpha}\right\rangle$.

\section{B. Composite interaction}

The detuning profile $\delta(t)$ used to stabilize $\left|\bar{c}_{\alpha}\right\rangle$ is represented on Fig. 11 (bottom part). The atomic frequency is first set at $\omega_{m}(\delta=0)$, between $t=-T / 2$ and $t=-t_{r}$. This corresponds to atoms which interact non-resonantly with both modes, the detuning w.r.t. mode $a$ being opposite to the detuning w.r.t. mode $b$. We restrict our analysis to the dispersive regime. The corresponding evolution 

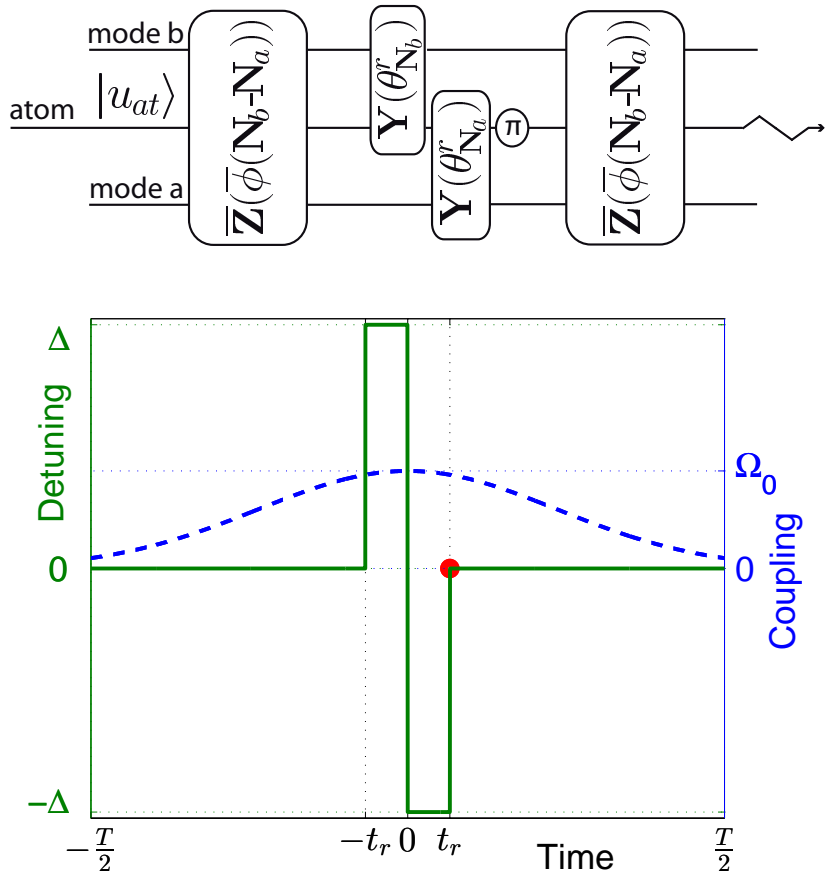

FIG. 11. (Color online) Schematic timing of the composite interaction of the atom with the two cavity modes $a$ and $b$ at frequencies $\omega_{b}>\omega_{a}$. Bottom frame, solid line: time profile of $\delta$ (difference between the atomic frequency $\omega_{0}$ and the mean frequency $\omega_{m}$ of the two cavity modes) during cavity crossing by one atomic sample. For $\delta$ values of $0,+\Delta$, and $-\Delta$ respectively, $\omega_{0}$ coincides with $\omega_{m}=\frac{\omega_{b}+\omega_{a}}{2}, \omega_{b}$, and $\omega_{a}$. The red dot represents a $\pi$ pulse acting on the atomic state. Bottom frame, dashed line: coupling strength $\Omega(v t)$, taking $t=0$ when the atom is at cavity center. Top frame: scheme of the propagators corresponding to the successive steps in the composite interaction.

operator is $\overline{\mathbf{Z}}\left(\bar{\phi}\left(\mathbf{N}_{b}-\mathbf{N}_{a}\right)\right.$ ) (see Appendix A), describing opposite phase shifts of the two modes driven by the atom, with a phase shift $\bar{\phi}$ per photon.

The atom is then successively set at resonance with modes $b$ and $a$ for a time $t_{r}$. During these short time intervals, we neglect the residual dispersive interaction with the other mode. A second dispersive interaction with the two modes is performed by setting $\delta=0$ again from $t=t_{r}$ to $t=T / 2$. With this sequence, the phase shifts produced during the two dispersive interactions would add up in the $|e\rangle\langle e|$-term of $\overline{\mathbf{U}}_{q}$, as in its $|g\rangle\langle g|$ term. Instead, we want these phase shifts to cancel out, like for the single-mode case. We therefore apply, at $t=t_{r}$, a $\pi$ rotation on the atom alone that swaps its levels $|g\rangle$ and $|e\rangle$. This rotation is driven by a classical source feeding a microwave pulse of negligible duration through the interval between the cavity mirrors. This pulse does not couple into the cavity modes.

The phases of modes $a$ and $b$ evolve at the frequencies $\pm \Delta$ w.r.t. a reference oscillator at the frequency $\omega_{m}$. In order to cancel the build-up of these phases during reservoir operation, we constrain the total time $T$ between successive interactions to satisfy $T \Delta=0$ modulo $2 \pi$. This can be achieved by an appropriately timing of the Stark shifts.

The propagator then writes (dropping irrelevant rotations; see Appendix A for a detailed calculation):

$$
\begin{aligned}
\overline{\mathbf{U}}_{T} \approx \overline{\mathbf{U}}_{\bar{c}}^{\mathrm{eff}}=\overline{\mathbf{Z}}\left(\bar{\phi}\left(\mathbf{N}_{b}-\mathbf{N}_{a}\right)\right) \overline{\mathbf{Y}}\left(\theta_{\mathbf{N}_{a}}^{r}\right) \\
\overline{\mathbf{Y}}\left(\theta_{\mathbf{N}_{b}}^{r}\right) \overline{\mathbf{Z}}\left(\bar{\phi}\left(\mathbf{N}_{a}-\mathbf{N}_{b}\right)\right) .
\end{aligned}
$$

By setting the dispersive interactions to produce a $\bar{\phi}=\pi$ phase shift per photon, we get

$$
\overline{\mathbf{U}}_{\bar{c}}^{\text {eff }}=e^{-i t_{K} \overline{\mathbf{H}}_{K}} \overline{\mathbf{Y}}\left(\theta_{\mathbf{N}_{a}}^{r}\right) \overline{\mathbf{Y}}\left(\theta_{\mathbf{N}_{b}}^{r}\right) e^{i t_{K} \overline{\mathbf{H}}_{K}},
$$

with $t_{K} \bar{\gamma}_{K}=\pi / 2$. Given the observations in Section VIIA and by developments strictly analogous to the single-mode case, the associated atomic reservoir thus stabilizes the entangled pointer state $\left|\bar{c}_{\alpha}\right\rangle$. Note that the detuning profile $\delta(t)$ can be adapted to obtain the same propagator when the interaction strength is not the same on both modes [43]. Generalization to entangled states with more than two coherent components in each mode is straightforward, using slightly more complex detuning sequences. Indeed each dispersive effect must then be implemented in two steps in order to induce identical instead of opposite phase-shifts on the two modes.

\section{Numerical simulations}

We numerically solve Eq. (42) and iterate the corresponding Kraus maps starting from the vacuum state. We select $u=\pi / 4$ and $\theta_{r}=\pi / 2$ such that the expected $\alpha$ is of the order of 1 . Decoherence is modeled by coupling each field mode with a separate thermal environment; both environments have the same damping time $T_{c}$ and the same temperature $\left(n_{t}=0.05\right)$. The interaction strength $\Omega(s)$ of the atom with each mode has the same Gaussian profile as in the single-mode case, with $\Omega_{0} / 2 \pi=50 \mathrm{kHz}$. The computations are run on a Hilbert space truncated to the 10 first Fock states for each field mode.

Figure 12 (solid line) shows the evolution of the fidelity $\left\langle\bar{c}_{\alpha}|\rho| \bar{c}_{\alpha}\right\rangle$ of the two-mode cavity state $\rho$ w.r.t. an entangled two-component MFSS $\left|\bar{c}_{\alpha}\right\rangle$, starting from the vacuum. We have chosen to illustrate the situation with $\Delta=8 \Omega_{0}, T_{c}=650 \mathrm{~ms}$. The atomic velocity is $v=22 \mathrm{~m} / \mathrm{s}$ and each atomic sample has a probability $p_{a t}=0.3$ to contain one interacting atom (and a probability 0.7 to contain none; here, we neglect samples eventually containing two atoms). The parameters of the reference $\left|\bar{c}_{\alpha}\right\rangle$, in particular $|\alpha|^{2}=0.67$, are numerically optimized to maximize the fidelity w.r.t. the reservoir stationary state $\left(\approx \rho_{200}\right)$. The engineered reservoir is efficient, since the optimal fidelity is $\approx 89 \%$. This value is reached after $\approx 30$ samples, corresponding to only 10 atoms on the average. To illustrate the protection of the 
state, we interrupt the reservoir after 200 atomic samples. As shown in Figure 12, the fidelity w.r.t. the target state then rapidly decreases.

The entangled nature of the state produced by the reservoir can be proven by showing that it violates a Bell inequality adapted to this two-mode case $[5,6]$. The tested Bell signal is:

$$
\begin{aligned}
\mathcal{B}\left(\gamma_{a}, \gamma_{b}, \gamma_{a}^{\prime}, \gamma_{b}^{\prime}\right)=\frac{\pi^{2}}{4} \mid \bar{W}\left(\gamma_{a}^{\prime}, \gamma_{b}^{\prime}\right)+\bar{W}\left(\gamma_{a}, \gamma_{b}^{\prime}\right) \\
+\bar{W}\left(\gamma_{a}^{\prime}, \gamma_{b}\right)-\bar{W}\left(\gamma_{a}, \gamma_{b}\right) \mid ;,
\end{aligned}
$$

where $\bar{W}\left(\gamma_{a}, \gamma_{b}\right)$ is the two-mode Wigner function. The latter is defined as:

$$
\bar{W}\left(\gamma_{a}, \gamma_{b}\right)=\frac{4}{\pi^{2}} \operatorname{Tr}\left(\mathbf{D}_{-\gamma_{a}}^{a} \mathbf{D}_{-\gamma_{b}}^{b} \rho \mathbf{D}_{\gamma_{a}}^{a} \mathbf{D}_{\gamma_{b}}^{b} \overline{\mathbf{P}}\right),
$$

where $\overline{\mathbf{P}}=e^{i \pi\left(\mathbf{N}_{a}+\mathbf{N}_{b}\right)}$ is a joint parity operator and $\mathbf{D}_{\gamma_{a}}^{a}$ and $\mathbf{D}_{\gamma_{b}}^{b}$ are the coherent displacement operators for modes $a$ and $b$ respectively. For separable states, compatible with a local realistic model, $\mathcal{B}$ is always smaller than 2. A value larger than 2 for a set of amplitudes $\left(\gamma_{a}, \gamma_{b}, \gamma_{a}^{\prime}, \gamma_{b}^{\prime}\right)$ is a proof that $\rho$ features quantum entanglement.

Figure 13 shows a cut of the two-mode Wigner function of $\rho_{200}$ in the plane $\operatorname{Re}\left(\gamma_{a}\right)=\operatorname{Re}\left(\gamma_{b}\right)=0$, in which maximum violation of the Bell inequality is expected [5]. A numerical maximization of the Bell signal $\mathcal{B}$ in this plane provides the four amplitudes shown as white dots. We have performed similar optimizations of $\mathcal{B}$ for each $\rho_{k}$ and plotted the maximum Bell signal $\mathcal{B}^{\max }$ as a dashed line in figure 12 . It reaches $\approx 2.1>2$ which implies that the reservoir stabilizes a provably entangled state of the modes. When the reservoir is switched off after 200 interactions, decoherence causes a rapid decrease of $\mathcal{B}^{\max }$.

Figure 14 shows the maximum Bell signal $\mathcal{B}^{\max }$ of the steady state as a function of $T_{c}$, for three detuning and atomic velocity values. The Bell inequality is violated for all these settings when $T_{c}>450 \mathrm{~ms}$. The curves crossing illustrates a competition between two effects. For small $T_{c}$ values, the Bell signal is larger when $\Delta$ is smaller, since a smaller $\Delta$ corresponds to a larger velocity and thus to more frequent atomic samples, separated by $T \approx 3 w / v$. The reservoir thus offers a stronger protection against decoherence when $\Delta$ is small. For large $T_{c}$, cavity damping becomes negligible w.r.t. the dispersive approximation error introduced in the reservoir action. In this case, we must choose a large $\Delta$ value in order to lower these approximation errors.

The $T_{c}$ values required for a Bell inequality violation are certainly difficult to reach, but they are only $\approx 3$ times larger than the best damping time reported so far [44]. The stabilization of such field states may thus be within reach of the next generation of experiments.

\section{CONCLUDING REMARKS}

We have proposed simple engineered reservoirs to stabilize a wide variety of non-classical field states in one

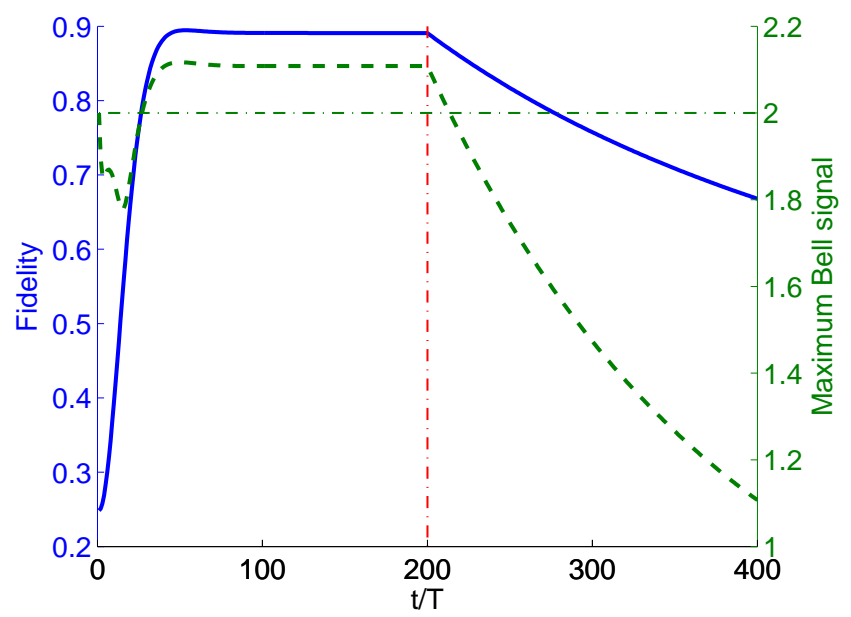

FIG. 12. (Color online) Simulation of the reservoir stabilizing a two-mode entangled state. Solid line: fidelity of $\rho$, the cavity state starting at vacuum, w.r.t. an ideal optimized entangled state of the two modes $\left|\bar{c}_{\alpha}\right\rangle$, as a function of time in units of the sample interaction time $T$. The reservoir operates up to $t / T=200$ and is then switched off. Dashed line: maximum Bell signal $\mathcal{B}^{\max }$ as a function of time. A $\mathcal{B}^{\max }$ value above the thin dash-dotted line $\left(\mathcal{B}^{\max }=2\right)$ proves entanglement of $\rho$.

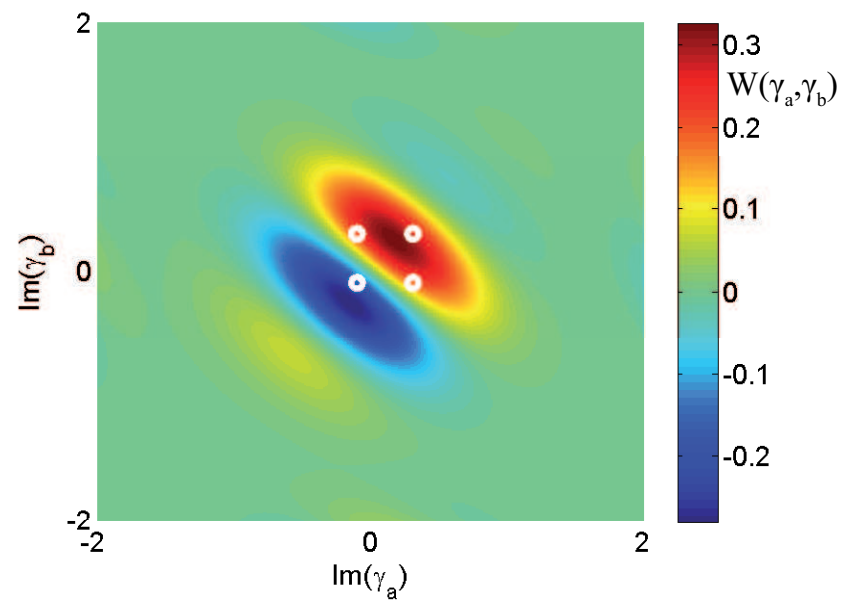

FIG. 13. (Color) Cut in the plane $\left(\operatorname{Re}\left(\gamma_{a}\right)=\operatorname{Re}\left(\gamma_{b}\right)=0\right)$ of the two-mode Wigner function $\bar{W}\left(\gamma_{a}, \gamma_{b}\right)$ of $\rho_{200}$. The fringes and negative values in $\bar{W}$ are a signature of the "quantumness" of the stabilized state. The white dots show the points that maximize the Bell signal (45).

and two cavity modes. These reservoirs efficiently counteract the standard relaxation of the cavities and offer promising perspectives for studies and applications of mesoscopic field state superpositions.

We have gained a detailed insight into the reservoir mechanisms, in particular how decoherence-induced quantum jumps of the field are corrected. We have performed extensive numerical simulations justifying the ap- 


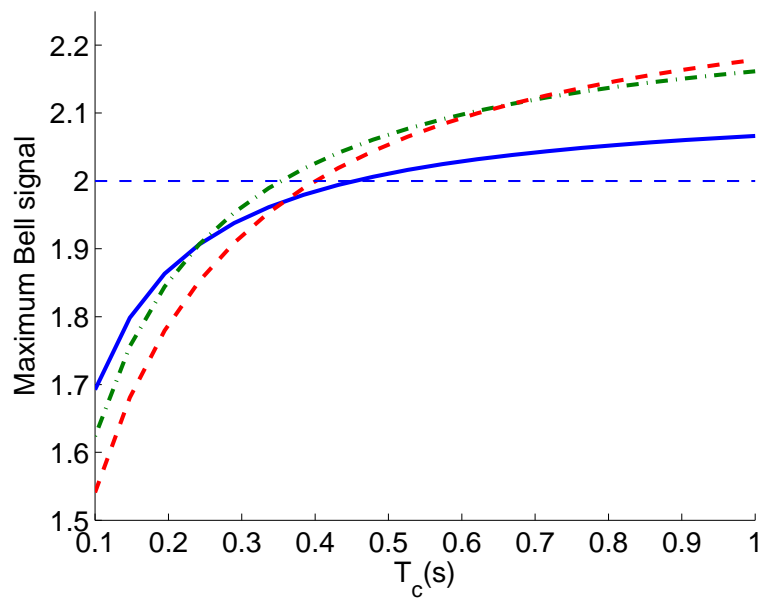

FIG. 14. (Color online) Maximum Bell signal $\mathcal{B}^{\max }$ of $\rho_{200}$ as a function of the cavity lifetime $T_{c}$, for $\Delta / 2 \pi=300 \mathrm{kHz}$, $v=30 \mathrm{~m} / \mathrm{s}$ (solid blue line); $\Delta / 2 \pi=400 \mathrm{kHz}, v=22 \mathrm{~m} / \mathrm{s}$ (dashed-dotted green line); $\Delta / 2 \pi=500 \mathrm{kHz}, v=18 \mathrm{~m} / \mathrm{s}$ (dashed red line).

proximations used in [32] and assessing the robustness of the method to experimental imperfections.

We have discussed here, for the sake of definiteness, the reservoir operation in the context of the microwaveCQED experiments performed with circular Rydberg atoms and superconducting cavities at ENS. We have shown that many quantum states protected by our reservoir could realistically be observed in this context. Clearly, the method could be straightforwardly extended to other spin/spring systems, in cavity QED and trapped-ions contexts. It is particularly appealing for the thriving field of circuit-QED [34]. Resettable superconducting qubits [33] interacting with one or two cavity modes could be used to implement our proposal. Making the qubits interact with two spatially separated cavities, it would become possible to stabilize a non-local entangled mesoscopic superposition and to study the fascinating interplay between decoherence and non-locality.

\section{Appendix A: Propagators}

This appendix details the computation of the propagators associated to the atom-cavity interaction for the various settings used in the main text.

\section{Single-mode case}

For a resonant interaction $[\delta(t)=0]$ between the field and an atom of velocity $v$ crossing the cavity axis at $t=0$, Eq. (4) writes:

$$
\frac{d}{d t} \mathbf{U}(t)=\frac{\Omega(s)}{2}\left(|g\rangle\left\langle e\left|\mathbf{a}^{\dagger}-\right| e\right\rangle\langle g| \mathbf{a}\right) \mathbf{U}(t)
$$

with $s=v t$. This interaction induces a Rabi rotation at an angular rate $\sqrt{n} \Omega(s)$ around the $Y$ axis of each Bloch sphere $B_{n}$ associated to the invariant space spanned by $(|g, n+1\rangle,|e, n\rangle)$. We therefore define the unitary operator $\mathbf{Y}\left(f_{\mathbf{N}}\right)[$ Eq. (9)] performing rotations around all these $Y$ axes in parallel by angles $f(n)$, where $f(n)$ is an arbitrary function of $n$. The resonant interaction propagator is then given by Eq. (12).

For an interaction between $t_{1}$ and $t_{2}$ with constant nonzero detuning $\delta(t)=\bar{\delta} \neq 0$ [45], the Gaussian variation of $\Omega(v t)$ precludes an exact integration of Eq. (4). However, assuming that $\Omega(v t)$ varies slowly enough, the coupled atom-field system evolves adiabatically. An initial eigenstate of $\mathbf{H}_{J C}\left(t_{1}\right)$ (a "dressed state") then remains, for any time $t$, close to an eigenstate of $\mathbf{H}_{J C}(t)$ [4]. This adiabatic approximation is valid provided:

$\left|\frac{2 v}{w \Omega_{0} \sqrt{n+1}} s e^{-s^{2}}\right| \ll\left(\frac{\bar{\delta}}{\Omega_{0} \sqrt{n+1}}\right)^{2}+e^{-2 s^{2}}, \quad \forall s \in\left(\frac{t_{1} v}{w}, \frac{t_{2} v}{w}\right)$,

for all $n$ in the relevant photon number range. This condition merely expresses that the interaction Hamiltonian $\mathbf{H}_{J C}$ varies slowly (through $\Omega(v t)$ ) in comparison to the differences between its eigenfrequencies.

The dressed states $\left(|-, n\rangle_{t},|+, n\rangle_{t}\right)$ that diagonalize the part of $\mathbf{H}_{J C}(t)$ acting on each $B_{n}$, for $n=1,2, \ldots$, satisfy

$$
\mathbf{H}_{J C}(t)| \pm, n\rangle_{t}= \pm \frac{\bar{\delta}}{2} \sqrt{1+(n+1)\left(\frac{\Omega(v t)}{\bar{\delta}}\right)^{2}}| \pm, n\rangle_{t}
$$

and write explicitly

$$
\begin{aligned}
|-, n\rangle_{t} & =\cos \left(\xi_{n}^{(t)} / 2\right)|g, n+1\rangle+i \sin \left(\xi_{n}^{(t)} / 2\right)|e, n\rangle \\
|+, n\rangle_{t} & =i \sin \left(\xi_{n}^{(t)} / 2\right)|g, n+1\rangle+\cos \left(\xi_{n}^{(t)} / 2\right)|e, n\rangle
\end{aligned}
$$

where we define $\xi_{n}^{(t)}$ by

$$
\tan \xi_{n}^{(t)}=\frac{\Omega(v t / 2) \sqrt{n}}{\bar{\delta}} \quad \text { with } \xi_{n}^{(t)} \in\left(\frac{-\pi}{2}, \frac{\pi}{2}\right) .
$$

The propagator $\mathbf{U}_{q}$ corresponding to the parameter set $q=\left(t_{1}, t_{2}, v, \bar{\delta}\right)$ is then

$$
\begin{aligned}
\mathbf{U}_{q}=\sum_{n}|-, n\rangle_{t_{2}}\langle- & ,\left.n\right|_{t_{1}} e^{\frac{i}{2} \phi_{n+1}^{q}} \\
& +|+, n\rangle_{t_{2}}\left\langle+,\left.n\right|_{t_{1}} e^{\frac{-i}{2} \phi_{n+1}^{q}},\right.
\end{aligned}
$$

where the accumulated phase $\phi_{n}^{q}$ is given by:

$$
\phi_{n}^{q}=\bar{\delta} \int_{t_{1}}^{t_{2}} \sqrt{1+n(\Omega(v t) / \bar{\delta})^{2}} d t
$$

from a full adiabatic propagator computation.

The restriction of $\mathbf{U}_{q}$ on the Bloch sphere $B_{n}$ can then be written as:

$$
\begin{aligned}
& |-, n\rangle_{t_{2}}\left\langle-,\left.n\right|_{t_{1}} e^{\frac{i}{2} \phi_{n+1}^{q}}+\mid+, n\right\rangle_{t_{2}}\left\langle+,\left.n\right|_{t_{1}} e^{\frac{-i}{2} \phi_{n+1}^{q}}\right. \\
& =\left(|-, n\rangle_{t_{2}}\langle g, n+1|+|+, n\rangle_{t_{2}}\langle e, n|\right) \\
& \times\left(|g, n+1\rangle\left\langle g, n+1\left|e^{\frac{i}{2} \phi_{n+1}^{q}}+\right| e, n\right\rangle\langle e, n| e^{\frac{-i}{2} \phi_{n+1}^{q}}\right) \\
& \times\left(|-, n\rangle_{t_{1}}\langle g, n+1|+|+, n\rangle_{t_{1}}\langle e, n|\right)^{\dagger} \text {. }
\end{aligned}
$$


The transformation $\left(|-, n\rangle_{t}\langle g, n+1|+|+, n\rangle_{t}\langle e, n|\right)$ is a rotation around the $X$-axis of $B_{n}$ by an angle $-\xi_{n+1}^{(t)}$. The transformation $\left(|g, n+1\rangle\langle g, n+1| e^{i \phi_{n+1}^{q} / 2}+\right.$ $\left.|e, n\rangle\langle e, n| e^{-i \phi_{n+1}^{q} / 2}\right)$ is a rotation around the $Z$-axis of $B_{n}$ by an angle $\phi_{n+1}^{q}$. We thus introduce in Eqs. (8),(10) the unitary operators $\mathbf{X}\left(f_{\mathbf{N}}\right)$ and $\mathbf{Z}\left(f_{\mathbf{N}}\right)$ performing such rotations in parallel on all the Bloch spheres $B_{n}$. Noting that $\mathbf{X}\left(-f_{\mathbf{N}}\right)^{\dagger}=\mathbf{X}\left(f_{\mathbf{N}}\right)$, we can finally write (A4) in the compact form:

$$
\mathbf{U}_{q}=\mathbf{X}\left(-\xi_{\mathbf{N}}^{\left(t_{2}\right)}\right) \mathbf{Z}\left(\phi_{\mathbf{N}}^{q}\right) \mathbf{X}\left(\xi_{\mathbf{N}}^{\left(t_{1}\right)}\right)
$$

At the start and end of the complete composite interaction in the main text, the atom-cavity coupling is weak: $\Omega^{2}( \pm v T / 2)=\Omega_{0}^{2} / 100$. Then $\xi_{\mathbf{N}}^{( \pm T / 2)} \approx 0$ and we can thus take as a good approximation $\mathbf{X}\left(-\xi_{\mathbf{N}}^{(-T / 2)}\right)=$ $\mathbf{X}\left(\xi_{\mathbf{N}}^{(T / 2)}\right)=\mathbf{I}$. This leads to Eq. (25) in Section V.

In the large detuning regime studied in Section IV, we can even neglect all the $\mathbf{X}$ operators in $\mathbf{U}_{q}$ compared to the large dispersive phase shift operator $\mathbf{Z}\left(\phi_{\mathbf{N}}^{q}\right)$.

\section{Two-mode case}

In the two-mode case, it is not possible to get an exact expression for the dressed states. We therefore assume a large detuning $2 \Delta$ between modes $a$ and $b$. We restrict moreover our analysis to two situations. First a (relatively short) resonant interaction with one of the modes alone. Second, a dispersive interaction with both modes. In the resonant case, we neglect the residual dispersive interaction with the other mode. For the non-resonant interaction, we use simple first-order dispersive expressions. For both cases, simulations explicitly integrating Eq. (42) confirm the validity of our approximations.

We define the following operators on the joint Hilbert space of the two field modes and the atom. For $k \in\{a, b\}$, $\overline{\mathbf{Y}}\left(\theta_{\mathbf{N}_{k}}^{q}\right)$ is the tensor product of the single-mode operator $\mathbf{Y}\left(\theta_{\mathbf{N}_{k}}^{q}\right)$ acting on the system (atom, mode $k$ ), with the identity acting on the other mode. A generalized twomode phase rotation is given by:

$$
\overline{\mathbf{Z}}\left(f_{\mathbf{N}_{a}, \mathbf{N}_{b}}\right)=|g\rangle\left\langle g\left|e^{\frac{i}{2} f_{\mathbf{N}_{a}, \mathbf{N}_{b}}}+\right| e\right\rangle\langle e| e^{\frac{-i}{2} f_{\left(\mathbf{N}_{a}+\mathbf{I}\right),\left(\mathbf{N}_{b}+\mathbf{I}\right)}},
$$

where the operator $f_{\mathbf{N}_{a}, \mathbf{N}_{b}}$ is diagonal in the joint Fock state basis of the two modes, with $f_{\mathbf{N}_{a}, \mathbf{N}_{b}}\left|n_{a}, n_{b}\right\rangle=$ $f\left(n_{a}, n_{b}\right)\left|n_{a}, n_{b}\right\rangle$.

First consider the resonant case, with $\delta= \pm \Delta$. A simple adaptation of the single mode result leads to:

$$
\begin{aligned}
\overline{\mathbf{U}}_{q}= & e^{-i \Delta\left(\mathbf{N}_{b}-\mathbf{N}_{a}\right)\left(t_{2}-t_{1}\right)} \overline{\mathbf{Z}}\left(\Delta\left(t_{2}-t_{1}\right)\right) \overline{\mathbf{Y}}\left(\theta_{\mathbf{N}_{b}}^{q}\right) \\
& \quad \text { for } q=\left(t_{1}, t_{2}, v, \Delta\right) \\
\overline{\mathbf{U}}_{q}= & e^{-i \Delta\left(\mathbf{N}_{b}-\mathbf{N}_{a}\right)\left(t_{2}-t_{1}\right)} \overline{\mathbf{Z}}\left(\Delta\left(t_{1}-t_{2}\right)\right) \overline{\mathbf{Y}}\left(\theta_{\mathbf{N}_{a}}^{q}\right) \\
& \quad \text { for } q=\left(t_{1}, t_{2}, v,-\Delta\right),
\end{aligned}
$$

where $\overline{\mathbf{Z}}$ defined by Eq. (A8) is here used with a constant argument $f_{\mathbf{N}_{a}, \mathbf{N}_{b}}= \pm \Delta\left(t_{2}-t_{1}\right)$.
Let us now consider the dispersive interaction used in the main text, corresponding to $\delta=0$. Applying second-order perturbation theory in $\Omega_{0} / \Delta$, we get for $q=\left(t_{1}, t_{2}, v, 0\right)$ :

$$
\overline{\mathbf{U}}_{q}=e^{-i \Delta\left(\mathbf{N}_{b}-\mathbf{N}_{a}\right)\left(t_{2}-t_{1}\right)} \overline{\mathbf{Z}}\left(\bar{\phi}^{q}\left(\mathbf{N}_{b}-\mathbf{N}_{a}\right)\right)
$$

with $\bar{\phi}^{q}=\frac{1}{2 \Delta} \int_{t_{1}}^{t_{2}} \Omega^{2}(v t) d t$.

Using Eqs. (A9),(A10),(A11) and the commutation relation (7), we get an approximate evolution operator for the sequence defined in Section VII, with $T \Delta=0$ modulo $2 \pi$ :

$$
\begin{aligned}
\overline{\mathbf{U}}_{T} \approx \overline{\mathbf{U}}_{\bar{c}}^{\mathrm{eff}}= & \overline{\mathbf{U}}_{\pi} \overline{\mathbf{Z}}\left(-\Delta\left(T / 2+t_{r}\right)\right) \\
& \overline{\mathbf{Z}}\left(\bar{\phi}\left(\mathbf{N}_{b}-\mathbf{N}_{a}\right)\right) \overline{\mathbf{Y}}\left(\theta_{\mathbf{N}_{a}}^{r}\right) \overline{\mathbf{Y}}\left(\theta_{\mathbf{N}_{b}}^{r}\right) \\
& \overline{\mathbf{Z}}\left(\bar{\phi}\left(\mathbf{N}_{a}-\mathbf{N}_{b}\right)\right) \overline{\mathbf{Z}}\left(-\Delta\left(T / 2-t_{r}\right)\right),
\end{aligned}
$$

where

$$
\bar{\phi}=\frac{1}{2 \Delta} \int_{-T / 2}^{-t_{r}} \Omega^{2}(v t) d t .
$$

The first line in Eq. (A12) has no effect on the Kraus map since it is a rotation on the atom only after it has interacted with the modes. The rightmost (i.e. first in time) operator $\overline{\mathbf{Z}}\left(-\Delta\left(T / 2-t_{r}\right)\right)$ can be compensated by properly setting the phase of the Ramsey pulse, preparing now each atom in $\overline{\mathbf{Z}}\left(\Delta\left(T / 2-t_{r}\right)\right)\left|u_{a t}\right\rangle$. These considerations lead to the effective propagator given in Eq. (44).

\section{Appendix B: Equilibrium of reservoir with damping}

We look for a stationary solution $\rho_{\infty}^{\prime h}$ to (38) under the form (39). Then we have:

$$
\begin{gathered}
\int_{-\alpha_{\infty}^{c}}^{\alpha_{\infty}^{c}} \mu(z)\left(\beta-\frac{\kappa+\kappa_{c}}{2} z\right)\left(\left(\mathbf{a}^{\dagger}-z\right)|z\rangle\langle z|+| z\rangle\langle z|(\mathbf{a}-z)\right) d z \\
+\int_{-\alpha_{\infty}^{c}}^{\alpha_{\infty}^{c}} \kappa_{c}(\mu(-z)-\mu(z)) z^{2}|z\rangle\langle z| d z=0,
\end{gathered}
$$

(using $\mathbf{a}|z\rangle=z|z\rangle, e^{i \pi \mathbf{N}} \mathbf{a}|z\rangle=z|-z\rangle$ and their Hermitian conjugates). Multiplying on the left by coherent state $\langle\xi|$ and on the right by $|\xi\rangle$, for any real $\xi$, yields

$$
\begin{aligned}
\int_{-\alpha_{\infty}^{c}}^{\alpha_{\infty}^{c}} 2 \mu(z)\left(\beta-\frac{\kappa+\kappa_{c}}{2} z\right)(\xi-z) e^{-(\xi-z)^{2}} d z \\
\quad+\int_{-\alpha_{\infty}^{c}}^{\alpha_{\infty}^{c}} \kappa_{c} z^{2}(\mu(-z)-\mu(z)) e^{-(\xi-z)^{2}} d z=0
\end{aligned}
$$


(recall that $|\langle\xi \mid z\rangle|^{2}=e^{-(\xi-z)^{2}}, \xi$ and $z$ being real). Applying integration by parts to the first term yields

$$
\begin{aligned}
& {\left[\mu(z)\left(\beta-\frac{\kappa+\kappa_{c}}{2} z\right) e^{-(\xi-z)^{2}}\right]_{z=-\alpha_{\infty}^{c}}^{z=\alpha_{\infty}^{c}}} \\
& \quad-\int_{-\alpha_{\infty}^{c}}^{\alpha_{\infty}^{c}}\left(\frac{d}{d z}\left(\mu(z)\left(\beta-\frac{\kappa+\kappa_{c}}{2} z\right)\right)\right) e^{-(\xi-z)^{2}} d z \\
& \quad+\int_{-\alpha_{\infty}^{c}}^{\alpha_{\infty}^{c}} \kappa_{c} z^{2}(\mu(-z)-\mu(z)) e^{-(\xi-z)^{2}} d z=0 .
\end{aligned}
$$

Since this holds for all real $\xi$, we get the condition:

$$
\kappa_{c} z^{2}(\mu(-z)-\mu(z))-\frac{d}{d z}\left(\mu(z)\left(\beta-\frac{\kappa+\kappa_{c}}{2} z\right)\right)=0
$$

for $z \in\left(-\alpha_{\infty}^{c}, \alpha_{\infty}^{c}\right)$ with boundary conditions $\lim _{z \mapsto \alpha_{\infty}^{c}} \mu(z)\left(z-\alpha_{\infty}^{c}\right)=0$ and $\mu\left(-\alpha_{\infty}^{c}\right)=0$.

To solve this differential equation for $z \in\left[-\alpha_{\infty}^{c}, \alpha_{\infty}^{c}\right]$, we decompose $\mu(z)$ in its even and odd parts. These parts satisfy two first-order coupled ordinary differential equa- tions that can be integrated directly to give expression (40) for $\mu(z)$.

\section{ACKNOWLEDGMENTS}

The authors thank S. Haroche, M. Mirrahimi and I. Dotsenko for enlightening discussions and references. AS has been an FNRS postdoctoral fellow at U. Liège, visiting researcher at Mines ParisTech and member of the IAP network DYSCO. ZL acknowledges support from Agence Nationale de la Recherche (ANR), Projet Jeunes Chercheurs EPOQ2 number ANR-09-JCJC-0070. JMR and MB acknowledge support from the EU and ERC (AQUTE and DECLIC projects). The authors were partially supported by the ANR, Projet Blanc EMAQS ANR2011-BS01-017-01, Projet Blanc QUSCO-INCA ANR09-BLAN-0123 and Projet C-QUID BLAN-3-139579.
[1] J.P. Dowling, Contemporary physics 49, 125 (2008).

[2] V. Giovannetti, S. Lloyd and L. Maccone, Science 306, 1330 (2004).

[3] K. Goda et al., Nature Physics 4, 472 (2008)

[4] S. Haroche and J.-M. Raimond. Exploring the Quantum: atoms, cavities and photons, Oxford University Press, Oxford (2006).

[5] K. Banaszek and K. Wódkiewicz, Phys. Rev. Lett 82, 2009 (1999).

[6] P. Milman et al., Eur. Phys. J. D 32, 233 (2005).

[7] B. Yurke and D. Stoler, Phys. Rev. Lett. 57, 13 (1986).

[8] S. Will et al., Nature(London) 465, 197 (2010).

[9] The interpretation as $k$ separated components only holds for $k<\pi \alpha$ [4, Section 7.2].

[10] M. Rosenbluh and R.M. Shelby, Phys. Rev. Lett. 66, 153 (1991).

[11] C. Monroe et al. Science 272, 1131 (1996).

[12] C.J. Myatt et. al. Nature(London) 403, 269 (2000).

[13] M. Brune et al., Phys. Rev. A 45, 5193 (1992).

[14] M. Brune et al., Phys. Rev. Lett. 77, 4887 (1996).

[15] S. Deléglise et al., Nature(London) 455, 510 (2008).

[16] L. Davidovich et al., Phys. Rev. Lett. 71, 2360 (1993).

[17] C.J. Villas-Bôas et al., J. Optics B 5, 391 (2003).

[18] E Solano, G.S. Agarwal and H. Walther, Phys. Rev. Lett. 90, 027903 (2003).

[19] R.L. de Matos Filho and W. Vogel, Phys. Rev. Lett. 76, 608 (1996).

[20] A. Ourjoumtsev et al., Nature(London) 448, 784 (2007).

[21] M. Brune et al. Phys. Rev. Lett. 77, 4887 (1996).

[22] W.H. Zurek, Phys. Rev. D 24, 1516 (1981).

[23] W.H. Zurek, Rev. Mod. Phys. 75, 715 (2003).

[24] J.F. Poyatos, J.I. Cirac and P. Zoller, Phys. Rev. Lett. 77, 4728 (1996).

[25] A.R.R. Carvalho et al., Phys. Rev. Lett. 86, 4988 (2001).

[26] J.T. Barreiro et al., Nature 470, 486-491 (2011).

[27] H. Krauter et al., Phys. Rev. Lett. 107, 080503 (2011).

[28] J.J. Slosser, P. Meystre and S.L. Braunstein, Phys. Rev.
Lett. 63, 934 (1989).

[29] G. Rempe, F. Schmidt-Kaler and H. Walther, Phys. Rev. Lett. 64, 2783 (1990).

[30] T. Werlang et al., Phys. Rev. A 78, 033820 (2008).

[31] S. Pielawa et al., Phys. Rev. Lett. 98, 240401 (2007).

[32] A. Sarlette et al., Phys. Rev. Lett. 107, 010402 (2011).

[33] M. D. Reed et al. , Appl. Phys. Lett. 96, 203110 (2010).

[34] M. H. Devoret and J.M. Martinis, Quantum Information Processing, 3, 163 (2004).

[35] K. Kraus. States, Effects and Operations: fundamental notions of quantum theory, Springer, Berlin (1983).

[36] F. Casagrande, A. Lulli and V. Santagostino, Phys. Rev. A 65, 023809 (2002).

[37] P. Filipovicz, J. Javanainen and P. Meystre, J. Opt. Soc. Am. B 3, 906 (1986).

[38] Explicitly, we have (all $|\cdot\rangle$ denote coherent states)

$$
\begin{aligned}
\epsilon \mathbf{N}|\beta\rangle & =|(1+\epsilon) \beta\rangle-|\beta\rangle+|\beta|^{2} \operatorname{Re}(\epsilon)|\beta\rangle \\
\epsilon \mathbf{a}^{\dagger}|\beta\rangle & =|\beta+\epsilon\rangle-|\beta\rangle+\operatorname{Re}\left(\epsilon \beta^{\dagger}\right)|\beta\rangle \\
\sum_{\nu} a_{\nu}\left|\beta+\epsilon_{\nu}\right\rangle & \left.=\sum_{\nu} a_{\nu}\right)\left|\frac{\sum_{\nu} a_{\nu}\left(\beta+\epsilon_{\nu}\right)}{\sum_{\nu} a_{\nu}}\right\rangle,
\end{aligned}
$$

up to second order terms in $|\epsilon|,\left|\epsilon_{\nu}\right|$, with $\epsilon, \epsilon_{\nu}, a_{\nu} \in \mathbb{C}$ and $\sum_{\nu} a_{\nu} \neq 0$.

[39] Note that $\chi_{n}^{c}$ is well-defined for all $n>0$ because $\left|\xi_{n}\right| \in$ $(0, \pi / 2)$. For $n=0$, the factor multiplying the undefined $e^{-i \phi_{0}^{c}}$ vanishes in Eq. (28).

[40] K. Molmer, Y. Castin and J. Dalibard, J. Opt. Soc. America B 10, 524 (1993).

[41] S.M. Tan, J. Opt. B 1, 424 (1999).

[42] A. Rauschenbeutel et al., Phys. Rev. A 64, 050301 (2001).

[43] Consider interaction strength $\Omega_{a}(s)=\Omega(s)$ with mode $a$ and $\Omega_{b}(s)=\lambda \Omega(s)$ with mode $b$, where $\lambda \neq 1$. Adjusting the resonant interaction times to get the same coherent state amplitude in both modes is straightforward. Equal dispersive phases are obtained by setting $\delta(t) \approx \bar{\delta}_{0}=$ $\Delta \frac{1-\lambda^{2}}{1+\lambda^{2}} \neq 0$ for $t \in\left(-T / 2,-t_{r} / 2\right) \cup\left(t_{r} / 2, T / 2\right)$. Indeed, 
to first order approximation in $\Omega_{0} / \Delta$, this choice shifts the eigenvalues of the Hamiltonian by equal amounts $\Omega_{a}(s) / \sqrt{1+\frac{\bar{\delta}_{0}}{\Delta}}=\Omega_{b}(s) / \sqrt{1-\frac{\bar{\delta}_{0}}{\Delta}}$ for the two modes.

[44] S. Kuhr et al. , Applied Physics Letters 90, 164101
(2007).

[45] The following developments also carry through with (slowly) continuously varying $\delta(t)$. For pedagogical reasons, we have chosen to explain the experiment with piecewise constant $\delta$. We therefore directly particularize the notation to $\delta(t)=\bar{\delta}$ constant. 